\title{
On the approximation of the unsteady Navier-Stokes equations by finite element projection methods
}

\author{
J.-L. Guermond ${ }^{1}$, L. Quartapelle ${ }^{2}$ \\ 1 Laboratoire d'Informatique pour la Mécanique et les Sciences de l'Ingénieur, CNRS, BP \\ 133, F-91403 Orsay, France; e-mail: guermond @limsi.fr \\ 2 Dipartimento di Fisica, Politecnico di Milano, Piazza Leonardo da Vinci, 32, \\ I-20133 Milano, Italy
}

Received October 2, 1995 / Revised version received July 9, 1997

Summary. This paper provides an analysis of a fractional-step projection method to compute incompressible viscous flows by means of finite element approximations. The analysis is based on the idea that the appropriate functional setting for projection methods must accommodate two different spaces for representing the velocity fields calculated respectively in the viscous and the incompressible half steps of the method. Such a theoretical distinction leads to a finite element projection method with a Poisson equation for the incremental pressure unknown and to a very practical implementation of the method with only the intermediate velocity appearing in the numerical algorithm. Error estimates in finite time are given. An extension of the method to a problem with unconventional boundary conditions is also considered to illustrate the flexibility of the proposed method.

Mathematics Subject Classification (1991): 35Q30, 65M12, 65M60

\section{Introduction}

The fractional-step projection method of Chorin [10,11] and Temam [28] (see also Temam [27] and Quartapelle [24]) is the most frequently employed technique for the numerical solution of the primitive variable Navier-Stokes equations. This method is based on a rather peculiar time-discretization of the equations governing viscous incompressible flows, in which the viscosity and the incompressibility of the fluid are dealt within two separate steps. The reader is referred to Rannacher [26] for a thorough analysis of the

Correspondence to: J.-L. Guermond

Numerische Mathematik Electronic Edition page 207 of Numer. Math. (1998) 80: 207-238 
convergence in time of the original Chorin-Temam algorithm (i.e. the nonincremental form); it is also shown in [26] that the projection algorithm can be interpreted as a pressure stabilization technique. In practice, the projection method is combined with any kind of spatial discretization technique, viz., finite differences (see e.g. Bell et al. [4]), finite elements (Donea et al. [13], Gresho and Chan [15]), or spectral approximations (Ku et al. [22]). The aim of the present paper to provide a framework and an error analysis for such fully discretized schemes.

An important, although almost never analyzed, feature of fractional-step projection methods is the structural difference existing between the equations of the viscous step and those of the incompressible phase of the calculation. In fact the first half-step constitutes an elliptic boundary value problem for an intermediate velocity unknown accounting for the viscous diffusion and convection mechanisms, whereas the second half-step represents an essentially inviscid problem that determines the end-of-step divergence-free velocity field together with a suitable approximation of the pressure distribution. In particular, boundary conditions of a different kind have to be imposed on the velocity fields that are calculated in each of the two halfsteps.

In spite of that, most (if not all) actual implementations of the projection method assume implicitly one and the same discrete representation for the two aforementioned velocity fields. But a single discretization cannot achieve the best approximation of the velocity for both the viscous and the inviscid phase of the method simultaneously. For instance, finite difference schemes based on staggered grids, such as the MAC computational molecule, are appropriate for representing the coupled equations of the inviscid projection step but are not the most convenient for discretizing the vector elliptic equation of the viscous step. Conversely, finite element discretizations using a continuous representation of the velocity by means of piecewise linear or multilinear polynomial interpolations are well suited for dealing with the viscous diffusion step but require a continuity degree higher than that required by the equations of the inviscid step.

A functional analytic setting that properly accounts for the different character of the equations of the two half-steps has been proposed recently by the first author $[16,17]$. The aim of this work is to provide a detailed analysis of projection methods that exploit the different mathematical structure of the equations for the two half-steps, as well as its consequences at the level of the spatially discretized equations. Insufficient consideration of this difference lies at the origin of the difficulties that the practical implementation of fractional-step projection methods is still encountering at present.

The content of the paper is organized as follows. In Sect. 2 the unsteady Navier-Stokes problem supplemented with Dirichlet boundary condition

Numerische Mathematik Electronic Edition page 208 of Numer. Math. (1998) 80: 207-238 
for velocity is first formulated in differential form. A variational statement of the problem is given and the abstract setting required for its spatial approximation by finite elements is finally introduced.

Section 3 describes an incremental version of the fractional-step time discretization of the Navier-Stokes equations and introduces the additional tools needed to have an abstract formulation of the spatially discrete equations of the two steps. Two possible numerical realizations of the equations enforcing incompressibility (the projection step) are considered, one regarding the projection step as a Darcy problem and the other as a Poisson problem for the pressure.

Section 4 addresses the Navier-Stokes equations in the presence of unconventional boundary conditions, comprising the specification of tangential components of vorticity and the imposition of boundary values of pressure. The role played by the pressure boundary conditions in the viscous and the incompressible steps is clearly indicated.

Section 5 details the error analysis of the proposed method for the case of Dirichlet conditions for the velocity. The last section is devoted to some concluding remarks.

\section{The unsteady Navier-Stokes problem}

\subsection{Hypotheses and notations}

Let $\Omega$ be an open connected bounded domain of $\mathbb{R}^{d}(d \leq 3)$ with a smooth boundary $\partial \Omega$. More specifically, the domain must be smooth enough so that Cattabriga's regularity estimates for the Stokes problem hold [9]; for instance, say $\partial \Omega$ is of class $C^{2}$ or $\Omega$ is a two-dimensional convex polygon. For more recent regularity estimates the reader is referred to Amrouche and Girault [1].

In the sequel, $W^{s, p}(\Omega)$ denotes the real Sobolev spaces, $0 \leq s<\infty, 0 \leq$ $p \leq \infty$, equipped with the norm $\|\cdot\|_{s, p}$ and semi-norm $|\cdot|_{s, p}$. The completion with respect to the $\|\cdot\|_{s, p}$ norm of the space of smooth functions compactly supported in $\Omega$ is denoted by $W_{0}^{s, p}(\Omega)$. The Hilbert spaces $W^{s, 2}(\Omega)$ (resp. $\left.W_{0}^{s, p}(\Omega)\right)$ is denoted by $H^{s}(\Omega)\left(\operatorname{resp} . H_{0}^{s}(\Omega)\right)$, the related norm is denoted by $\|\cdot\|_{s}$, and the dual space of $H_{0}^{s}(\Omega)$ is denoted by $H^{-s}(\Omega)$.

We consider the following time-dependent Navier-Stokes problem in which homogeneous Dirichlet condition has been assumed for simplicity. For a given body force $f$ (possibly dependent on time) and a given divergence-free initial velocity field $u_{0}$, find a velocity field $u$ and a pressure field $p$ (with regularities yet to be clearly defined) so that at $t=0$,

Numerische Mathematik Electronic Edition page 209 of Numer. Math. (1998) 80: 207-238 
$u=u_{0}$, and at all subsequent times

$$
\left\{\begin{array}{l}
\frac{\partial u}{\partial t}-\nabla^{2} u+(u \cdot \nabla) u+\nabla p=f, \\
\nabla \cdot u=0
\end{array}\right.
$$

the velocity being subject to the homogeneous Dirichlet condition

$$
u_{\mid \partial \Omega}=0 .
$$

Other types of boundary conditions are considered in Sect. 4.

\subsection{The variational formulation}

To formulate the unsteady Navier-Stokes problem in a variational form, we introduce the following Hilbert spaces:

$$
\begin{aligned}
& X=H_{0}^{1}(\Omega)^{d}, \quad M=L_{0}^{2}(\Omega)=\left\{q \in L^{2}(\Omega), \int_{\Omega} q=0\right\} \\
& V=\{v \in X, \nabla \cdot v=0\} \\
& H=\left\{v \in L^{2}(\Omega)^{d}, \nabla \cdot v=0, v \cdot n_{\mid \partial \Omega}=0\right\}
\end{aligned}
$$

The importance of $H$ is emphasized by the following classical orthogonal decomposition of $L^{2}(\Omega)^{d}$, the discrete counterpart of which will play a key role in the projection technique to be presented in Sect. 3,

$$
L^{2}(\Omega)^{d}=H \oplus \nabla\left(H^{1}(\Omega)\right) .
$$

To simplify the notations and express problem (2.1) in an variational framework we define the linear continuous operator $A: X \longrightarrow X^{\prime}$ (resp. bilinear form $\left.a: X^{2} \longrightarrow \mathbb{R}\right)$ so that for all $(u, v) \in X \times X$ :

$$
\langle A u, v\rangle=a(u, v)=(\nabla u, \nabla v)=\sum_{i, j=1}^{d}\left(\frac{\partial u_{i}}{\partial x_{j}}, \frac{\partial v_{i}}{\partial x_{j}}\right) .
$$

We also introduce the operator $B: X \longrightarrow M$ and its transpose $B^{\mathrm{T}}: M \longrightarrow$ $X^{\prime}$ (resp. linear form $b: X \times M \longrightarrow \mathbb{R}$ ) so that for all $v \in X$ and $q \in M$,

$$
(B v, q)=b(v, q)=-(\nabla \cdot v, q)
$$

The nonlinear term is taken into account through the bilinear operator $D: X^{2} \longrightarrow X^{\prime}$ (resp. trilinear form $d: X^{3} \longrightarrow \mathbb{R}$ ) so that

$$
\begin{aligned}
& \forall(u, v, w) \in X^{3}, \\
& \langle D(u, v), w\rangle=d(u, v, w)=((u \cdot \nabla) v, w)+\frac{1}{2}(\nabla \cdot u, v \cdot w),
\end{aligned}
$$

where $u \cdot v$ denotes the Euclidean scalar product in $\mathbb{R}^{d}$. Note that $D(u, v)$ coincides with the usual $(u \cdot \nabla) v$ form when $u$ is divergence free. By using the

Numerische Mathematik Electronic Edition page 210 of Numer. Math. (1998) 80: 207-238 
Hölder inequality together with some Sobolev inequalities (cf. e.g. Brezis [7]), we obtain the following bounds which will be used repeatedly hereafter:

$$
\max (d(u, v, w), d(v, u, w)) \leq c\left(\|u\|_{0, \infty}+\|u\|_{1,3}\right)\|v\|_{0,2}\|w\|_{1,2} .
$$

We recall also that the Gagliardo-Nirenberg [7] interpolation inequalities yield

$$
\|u\|_{0, \infty}+\|u\|_{1,3} \leq c\|u\|_{1,2}^{1 / 2}\|u\|_{2,2}^{1 / 2} .
$$

For a given $f \in W^{2, \infty}\left(0, \infty ; L^{2}(\Omega)^{d}\right)$ and a given initial velocity field $u_{0} \in V \cap H^{2}(\Omega)^{d}$, the variational formulation of (2.1) consists in finding a pair $(u, p)$

$u \in L^{\infty}(0, T ; H) \cap L^{2}(0, T ; V), u_{t} \in L^{2}\left(0, T ; H^{-1}\right), \quad$ for all $T>0$, $p \in L^{2}(0, T ; M), \quad$ for all $T>0$,

so that

$$
\left\{\begin{array}{l}
u_{t}+A u+D(u, u)+B^{\mathrm{T}} p=f, \\
B u=0, \\
u(0)=u_{0} .
\end{array}\right.
$$

In the following, we shall assume that (2.10) has a unique solution and that this solution is as smooth as needed. Furthermore, we shall assume that the data satisfy all the compatibility conditions required for a smooth solution in time to exist.

\subsection{The spatial discretization}

Let $\mathcal{T}_{h}$ be a regular, quasi-uniform triangulation of $\Omega$. We introduce $X_{h}$ and $M_{h}$ a mixed finite element approximations of $X$ and $M$ based on the triangulation $\mathcal{T}_{h}$. It is assumed hereafter that the following properties hold (see e.g. Bernardi and Raugel [6], Girault and Raviart [14], or Quarteroni and Valli [25] for other details):

There is $l \geq 1$ and $c>0$ such that for all $r(0 \leq r \leq l)$

$$
\begin{aligned}
\inf _{v_{h} \in X_{h}}\left\{\left\|v-v_{h}\right\|_{0}+h\left\|v-v_{h}\right\|_{1}\right\} \leq c h^{r+1}\|v\|_{r+1}, & \\
\forall v & \in H^{r+1}(\Omega)^{d} \cap X, \\
\inf _{v_{h} \in X_{h}}\left\|v-v_{h}\right\|_{1, p} \leq c h^{r}\|v\|_{r+1, p}, & \leq p \leq \infty, \\
\forall v & \in W^{r+1, p}(\Omega)^{d} \cap X .
\end{aligned}
$$

There exists $c>0$ such that for all $r(0 \leq r \leq l)$ and for all $q$ in $H^{r}(\Omega) \cap M$,

$$
\inf _{q_{h} \in M_{h}}\left\|q-q_{h}\right\|_{0} \leq c h^{r}\|q\|_{r}
$$

Numerische Mathematik Electronic Edition page 211 of Numer. Math. (1998) 80: 207-238 
There is $c>0$ such that for all $v_{h}$ in $X_{h}$, the following inverse inequalities hold:

$$
\begin{aligned}
& \left\|v_{h}\right\|_{n, p} \leq c h^{m-n+\frac{d}{p}-\frac{d}{q}}\left\|v_{h}\right\|_{m, q}, \\
& 0 \leq m \leq n \leq 1, \quad 1 \leq q \leq p \leq \infty .
\end{aligned}
$$

We introduce the linear operator $B_{h}: X_{h} \longrightarrow M_{h}$ and its transpose $B_{h}^{\mathrm{T}}: M_{h} \longrightarrow X_{h}$ so that for every couple $\left(v_{h}, q_{h}\right)$ in $X_{h} \times M_{h}$ we have $\left(B_{h} v_{h}, q_{h}\right)=b\left(v_{h}, q_{h}\right)$ and $\left(v_{h}, B_{h}^{\mathrm{T}} q_{h}\right)=b\left(v_{h}, q_{h}\right)$. We assume in the sequel that $B_{h}$ is surjective; that is to say, the mixed approximation satisfies the Babǔska-Brezzi condition, [3] [8]:

$$
\exists c>0, \quad \inf _{q_{h} \in M_{h}} \sup _{v_{h} \in X_{h}} \frac{\left(B_{h} v_{h}, q_{h}\right)}{\left\|v_{h}\right\|_{1}\left\|q_{h}\right\|_{0}} \geq c .
$$

Let us also introduce the linear continuous operator $A_{h}: X_{h} \longrightarrow X_{h}$ so that, for all $\left(u_{h}, v_{h}\right) \in X_{h} \times X_{h},\left(A_{h} u_{h}, v_{h}\right)=a\left(u_{h}, v_{h}\right)$. The discrete advection operator is defined by $\left(D_{h}\left(u_{h}, v_{h}\right), w_{h}\right)=d\left(u_{h}, v_{h}, w_{h}\right)$. Note that even if $u_{h}$ is not divergence free, the bilinear form $d\left(u_{h}, \cdot, \cdot\right)$ is skewsymmetric: $d\left(u_{h}, v_{h}, w_{h}\right)=-d\left(u_{h}, w_{h}, v_{h}\right)$; as a result $d\left(u_{h}, v_{h}, v_{h}\right)=0$. Finally, we introduce $i_{X_{h}}: X_{h} \longrightarrow X$ the continuous injection of $X_{h}$ into $X$, and $i_{X_{h}}^{\mathrm{T}}$ its transpose.

In the functional framework defined above, the Galerkin spatial approximation of (2.10) based on $\left(X_{h}, M_{h}\right)$ is formulated as follows. Find $u_{h} \in H^{1}\left(0, T ; X_{h}\right)$ and $p_{h} \in L^{2}\left(0, T ; M_{h}\right)$ so that:

$$
\left\{\begin{array}{l}
u_{h, t}+A_{h} u_{h}+D_{h}\left(u_{h}, u_{h}\right)+B_{h}^{\mathrm{T}} p_{h}=i_{X_{h}}^{\mathrm{T}} f, \\
B_{h} u_{h}=0 \\
u_{h \mid t=0}=u_{0, h} .
\end{array}\right.
$$

where $u_{0, h}$ is an approximation of $u_{0}$ in $\operatorname{ker}\left(B_{h}\right)$. The discrete counterpart of the body force is hereafter denoted by $f_{h}$ for simplicity. Problem (2.15) is well posed and it is possible to show that $\left(u_{h}(t), p_{h}(t)\right)$ converges in some appropriate sense to the solution of (2.10) (cf. Heywood and Rannacher [21]). In the following we are interested in approximating the time-dependent problem (2.15) by means of a projection technique for $t \geq t_{0}>0$.

\section{The fractional-step projection algorithms}

\subsection{The discrete setting}

The functional framework defined above is suitable for classical approximations of the Navier-Stokes problem as a system of coupled equations; however, aiming at uncoupling the incompressibility constraint from the

Numerische Mathematik Electronic Edition page 212 of Numer. Math. (1998) 80: 207-238 
time evolution problem, we are led to introduce additional tools (see [16, 17] for other details). We define $Y_{h}$ a finite dimensional subspace of $L^{2}(\Omega)^{d}$ and endow $Y_{h}$ with the norm of $L^{2}(\Omega)^{d}$; for the sake of simplicity we assume that $X_{h} \subset Y_{h}$ (in terms of vector space) and we denote by $i_{h}$ the continuous injection of $X_{h}$ into $Y_{h}$; the transpose of $i_{h}$ is the $L^{2}$ projection of $Y_{h}$ onto $X_{h}$. Note that $Y_{h}$ is an internal approximation of $L^{2}(\Omega)^{d}$, for $X_{h}$ is an approximation of $X$ and $X$ is dense in $L^{2}(\Omega)^{d}$. Furthermore, we assume that $Y_{h}$ and $M_{h}$ are compatible in the sense that either $Y_{h}$ is conformal in

$$
H_{0}^{\operatorname{div}}(\Omega)=\left\{v \in L^{2}(\Omega)^{d}, \nabla \cdot v \in L^{2}(\Omega), v \cdot n_{\mid \partial \Omega}=0\right\}
$$

or $M_{h}$ is conformal in $H^{1}(\Omega)$. For instance, we have the trivial choice $Y_{h}=X_{h}$, but we can also choose $Y_{h} \subset H_{0}^{\operatorname{div}}(\Omega)$; another interesting choice is $Y_{h} \subset L^{2}(\Omega)^{d}$ and $M_{h} \subset H^{1}(\Omega)$ (see further below and [16,17] for other details).

The analysis of the fractional-step equations in spatially discrete form requires to introduce another discrete version of the divergence operator. Let $C_{h}: Y_{h} \longrightarrow M_{h}$ be so that for every couple $\left(v_{h}, q_{h}\right)$ in $Y_{h} \times M_{h}$, either $\left(C_{h} v_{h}, q_{h}\right)=-\left(\nabla \cdot v_{h}, q_{h}\right)$ if $Y_{h} \subset H_{0}^{\operatorname{div}}(\Omega)$ or $\left(C_{h} v_{h}, q_{h}\right)=\left(v_{h}, \nabla q_{h}\right)$ if $M_{h} \subset H^{1}(\Omega)$. Of course this definition makes sense given the compatibility we require between $Y_{h}$ and $M_{h}$. The relation between $B_{h}$ and $C_{h}$ is brought to light by

Proposition 3.1. $C_{h}$ is an extension of $B_{h}$ and $i_{h}^{\mathrm{T}} C_{h}^{\mathrm{T}}=B_{h}^{\mathrm{T}}$; in other words we have the following commutative diagrams:
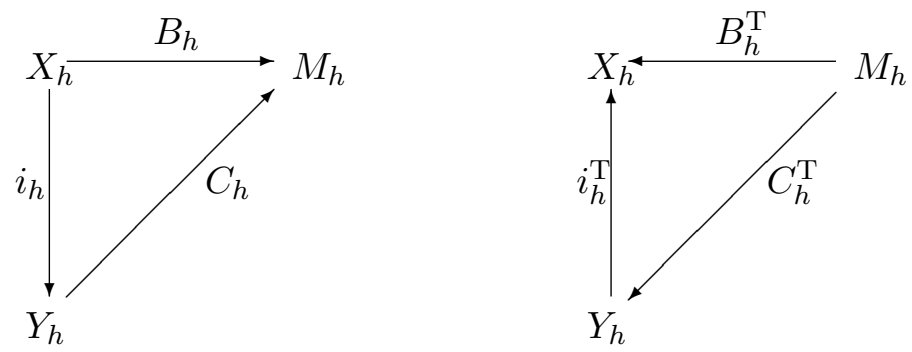

Proof. (a) Assume $Y_{h} \subset H_{0}^{\operatorname{div}}(\Omega)$. For all $\left(v_{h}, q_{h}\right)$ in $X_{h} \times M_{h}$, we have $\left(C_{h} i_{h} v_{h}, q_{h}\right)=-\left(\nabla \cdot\left(i_{h} v_{h}\right), q_{h}\right)=-\left(\nabla \cdot v_{h}, q_{h}\right)=\left(B_{h} v_{h}, q_{h}\right)$ since $X_{h} \subset Y_{h}$; that is, $C_{h} i_{h} v_{h}=B_{h} v_{h}$ for all $v_{h} \in X_{h}$.

(b) Assume $M_{h} \subset H^{1}(\Omega)$. For all $\left(v_{h}, q_{h}\right)$ in $X_{h} \times M_{h}$, we have $\left(C_{h} i_{h} v_{h}, q_{h}\right)=\left(i_{h} v_{h}, \nabla q_{h}\right)=\left(v_{h}, \nabla q_{h}\right)=-\left(\nabla \cdot v_{h}, q_{h}\right)=\left(B_{h} v_{h}, q_{h}\right)$ since $X_{h} \subset Y_{h}$; that is, $C_{h} i_{h} v_{h}=B_{h} v_{h}$ for all $v_{h} \in X_{h}$.

(c) By taking the transpose of $C_{h} i_{h}=B_{h}$ we obtain $i_{h}^{\mathrm{T}} C_{h}^{\mathrm{T}}=B_{h}^{\mathrm{T}}$.

Recall that $B_{h}$ is assumed to be surjective; as a consequence, $C_{h}$ is also surjective for $C_{h}$ is an extension of $B_{h}$. The null space of $C_{h}$ playing an important role in the sequel, we set $H_{h}=\operatorname{ker} C_{h}$. This definition

Numerische Mathematik Electronic Edition page 213 of Numer. Math. (1998) 80: 207-238 
yields a discrete counterpart of the aforementioned orthogonal decomposition $L^{2}(\Omega)^{d}=H \oplus \nabla\left(H^{1}(\Omega)\right)$.

Corollary 3.2. The following orthogonal decomposition of $Y_{h}$ holds:

$$
Y_{h}=H_{h} \oplus C_{h}^{\mathrm{T}}\left(M_{h}\right) \text {. }
$$

\subsection{The fractional-step projection scheme}

For a fixed finite time $T>0$, introduce a partition of the time interval $[0, T]$ : $t^{k}=k \delta t$ for $0 \leq k \leq K$ where $\delta t=T / K$. To avoid the technical difficulty of the possible blow up of the estimates at the initial time induced by the possible lack of regularity of the continuous solution, one may assume that the time dependent Galerkin problem (2.15) is approximated in time by an implicit (or semi-implicit, if the advection term is linearized) Euler scheme of first order from $k=1$ to some $k_{0}\left(1<k_{0}<K\right)$ so that $t_{0}=k_{0} \delta t$ is some fixed time independent of $K$. Denote by $\hat{u}_{h}^{k_{0}}$ and $\hat{p}_{h}^{k_{0}}$ the approximate solution at time step $t^{k_{0}}$; given the smoothing properties of the Navier-Stokes equations it can be shown that

$$
\left\{\begin{array}{l}
\left\|u\left(t^{k_{0}}\right)-\hat{u}_{h}^{k_{0}}\right\|_{0} \leq c\left(h^{l+1}+\delta t\right), \\
\left\|u\left(t^{k_{0}}\right)-\hat{u}_{h}^{k_{0}}\right\|_{1}+\left\|p\left(t^{k_{0}}\right)-\hat{p}_{h}^{k_{0}}\right\|_{0} \leq c\left(h^{l}+\delta t\right) .
\end{array}\right.
$$

For an exhaustive error analysis of this type of approximation the reader is referred to Heywood and Rannacher [21]; see also Bernardi and Raugel [6].

We are now interested in defining a projection scheme for $k_{0} \leq k \leq K$; define two sequences of approximate velocities $\left\{\tilde{u}_{h}^{k} \in X_{h}\right\}$ and $\left\{u_{h}^{k} \in Y_{h}\right\}$ and one sequence of approximate pressures $\left\{p_{h}^{k} \in M_{h}\right\}$ so that

$$
\frac{\tilde{u}_{h}^{k+1}-i_{h}^{\mathrm{T}} u_{h}^{k}}{\delta t}+A_{h} \tilde{u}_{h}^{k+1}+D_{h}\left(\tilde{u}_{h}^{k}, \tilde{u}_{h}^{k+1}\right)=f_{h}^{k+1}-B_{h}^{\mathrm{T}} p_{h}^{k}
$$

and

$$
\left\{\begin{array}{l}
\frac{u_{h}^{k+1}-i_{h} \tilde{u}_{h}^{k+1}}{\delta t}+C_{h}^{\mathrm{T}}\left(p_{h}^{k+1}-p_{h}^{k}\right)=0 \\
C_{h} u_{h}^{k+1}=0
\end{array}\right.
$$

The sequences $\left\{u_{h}^{k}\right\}$ and $\left\{\tilde{u}_{h}^{k}\right\}$ are initialized by $u_{h}^{k_{0}}=\tilde{u}_{h}^{k_{0}}=\hat{u}_{h}^{k_{0}}$ and the sequence $\left\{p_{h}^{k}\right\}$ is initialized by $p_{h}^{k_{0}}=\hat{p}_{h}^{k_{0}}$.

Remark 3.1. The problem (3.3) is well posed since, given the skew-symmetry of $d$, the linear operator $A_{h}(\cdot)+D_{h}\left(\tilde{u}_{h}^{k}, \cdot\right)$ is $X$-elliptic. The problem (3.4) is also well posed thanks to Corollary 3.2: indeed the couple $\left(u_{h}^{k+1}\right.$, $\left.\delta t C_{h}^{\mathrm{T}}\left(p_{h}^{k+1}-p_{h}^{k}\right)\right)$ is the decomposition of $i_{h} \tilde{u}_{h}^{k+1}$ in $H_{h} \oplus C_{h}^{\mathrm{T}}\left(M_{h}\right)$; in other words, $u_{h}^{k+1}=P_{H_{h}}\left(i_{h} \tilde{u}_{h}^{k+1}\right)$, where $P_{H_{h}}$ is the operator of orthogonal projection of $Y_{h}$ onto $H_{h}$.

Numerische Mathematik Electronic Edition page 214 of Numer. Math. (1998) 80: 207-238 
Remark 3.2. The original Chorin-Temam algorithm does not contain $B_{h}^{\mathrm{T}} p_{h}^{k}$ in the right-hand side of (3.3) and contains only the unknown $p_{h}^{k+1}$ in (3.4); in the sequel we refer to it as the nonincremental algorithm, whereas the algorithm presented here is referred to as the incremental one. Most of what is said in this paper applies also to the original nonincremental projection algorithm. In short, both algorithms converge, but the incremental one has a better rate of convergence than the nonincremental one in the natural norms (see Guermond [16], [18], and Rannacher [26]).

In practice it is not very convenient to solve the problem as presented here. Actually, the projected velocity $u_{h}^{k}$ can be eliminated from the algorithm as follows (see Guermond and Quartapelle [19]). Replace $u_{h}^{k}$ in (3.3) by its definition which is given by (3.4) at the time step $t^{k}$; note that $i_{h}^{\mathrm{T}} C_{h}^{\mathrm{T}}=B_{h}^{\mathrm{T}}$, as already mentioned. In (3.4), $u_{h}^{k+1}$ is eliminated by applying $C_{h}$ to the first equation and by noting that $C_{h}$ is an extension of $B_{h}$. The algorithm that should be implemented reads, for $k \geq k_{0}+1$,

$$
\begin{aligned}
\frac{\tilde{u}_{h}^{k+1}-\tilde{u}_{h}^{k}}{\delta t}+A_{h} \tilde{u}_{h}^{k+1}+ & D_{h}\left(\tilde{u}_{h}^{k}, \tilde{u}_{h}^{k+1}\right) \\
& =f_{h}^{k+1}-B_{h}^{\mathrm{T}}\left(2 p_{h}^{k}-p_{h}^{k-1}\right)
\end{aligned}
$$

and

$$
C_{h} C_{h}^{\mathrm{T}}\left(p_{h}^{k+1}-p_{h}^{k}\right)=\frac{B_{h} \tilde{u}_{h}^{k+1}}{\delta t} .
$$

For $k=k_{0}$ one step of the incremental algorithm in the original form (3.3)-(3.4) is performed.

The description of the algorithm above is somewhat abstract; we now show its practical implementation in two different contexts.

\subsection{The projection step as a Darcy problem}

To be more explicit, we show how the method should be implemented in the particular situation $Y_{h}=X_{h}$. In such a case no distinction is needed between the operators $B_{h}$ and $C_{h}$. For an example of practical implementation of this framework, the reader is referred to Gresho and Chan [15].

The viscous step amounts to looking for $\tilde{u}_{h}^{k+1}$ in $X_{h}$ so that, for all $v_{h} \in X_{h}$

$$
\text { (3.7) } \begin{aligned}
\left(\frac{\tilde{u}_{h}^{k+1}-u_{h}^{k}}{\delta t}, v_{h}\right)+a\left(\tilde{u}_{h}^{k+1}, v_{h}\right)+ & d\left(\tilde{u}_{h}^{k}, \tilde{u}_{h}^{k+1}, v_{h}\right) \\
& =\left(f^{k+1}, v_{h}\right)+\left(p_{h}^{k}, \nabla \cdot v_{h}\right) .
\end{aligned}
$$

Numerische Mathematik Electronic Edition page 215 of Numer. Math. (1998) 80: 207-238 
The projection step consists in looking for $u_{h}^{k+1}$ in $X_{h}$ and $p_{h}^{k+1}$ in $M_{h}$ so that

$$
\begin{cases}\forall v_{h} \in X_{h}, & \left(\frac{u_{h}^{k+1}-\tilde{u}_{h}^{k+1}}{\delta t}, v_{h}\right)-\left(\left(p_{h}^{k+1}-p_{h}^{k}\right), \nabla \cdot v_{h}\right)=0 \\ \forall q_{h} \in M_{h}, & \left(\nabla \cdot u_{h}^{k+1}, q_{h}\right)=0\end{cases}
$$

Remark 3.3. Note that in the projection step the velocity $u_{h}^{k+1}$ is sought in $X_{h}$. As a result, $u_{h}^{k+1}$ satisfies the essential boundary conditions enforced by $X_{h}$. In some sense this may seem surprising, even paradoxical, for the projection step should be the discrete counterpart of the continuous one, in which the projected velocity belongs to $H$, and therefore must satisfy the boundary condition $u \cdot n_{\mid \partial \Omega}=0$. Indeed, there is no contradiction for, $X$ being dense in $H_{0}^{\operatorname{div}}(\Omega)$, any approximation $X_{h}$ of $X$ is also an approximation of $H_{0}^{\text {div }}(\Omega)$; that is, the projected velocity can be perfectly approximated by elements of $X_{h}$. As a matter of facts, problem (3.8) can be understood as a discrete approximation of a Darcy problem.

Remark 3.4. It is shown in [17] that this approximation is optimal for finite element spatial discretizations in the sense that the pressure operator associated with the projection step has an optimal condition number. This result is likely to be no longer true when spectral approximations are used. Optimality can be recovered if (3.4) is reformulated with test functions satisfying $v \cdot n_{\mid \partial \Omega}=0$. This can be done by choosing $Y_{h}$ as a subspace of $H_{0}^{\operatorname{div}}(\Omega)$ and seeking $u_{h}^{k+1}$ in $Y_{h}$, which means to enforce only $u_{h}^{k+1} \cdot n_{\mid \partial \Omega}=0$. This technique is optimal for spectral approximations (see [2] for examples of approximations of this kind), since for some cases of spectral approximations it is possible to show that the pressure operator $C_{h} C_{h}^{\mathrm{T}}$ is better conditioned than $B_{h} B_{h}^{\mathrm{T}}$. Indeed this approach constitutes a second possible implementation. We just mention it and shall not dwell on this matter (see $[16,17]$ for other details). For finite element approximations the condition numbers of both operators are equivalent. As a result, the considered implementation, with $Y_{h}=X_{h}$ and $C_{h}=B_{h}$, may be recommended for finite element discretizations, one advantage of such an approach being that the operators (and also the matrices) $B_{h}$ and $C_{h}$ involved in (3.3) and (3.4) are identical.

In practice we do not manipulate operators but matrices; that is, we choose particular bases of $X_{h}$ and $M_{h}$. Each of these choices yields a consistent mass matrix $\mathcal{I}_{h}$ and a matrix associated with the divergence operator, say $\mathcal{B}_{h}$. For a velocity field $u_{h}$ in $X_{h}$ and a pressure field $p_{h}$ in $M_{h}$, we denote by $U$ and $P$ the vectors of the components of $u_{h}$ and $p_{h}$ in the bases in question. In this context, the projection step described above yields the

Numerische Mathematik Electronic Edition page 216 of Numer. Math. (1998) 80: 207-238 
following linear system in terms of the pressure unknowns

$$
\mathcal{B}_{h} \mathcal{I}_{h}^{-1} \mathcal{B}_{h}^{\mathrm{T}}\left(P^{k+1}-P^{k}\right)=\frac{\mathcal{B}_{h} \tilde{U}^{k+1}}{\delta t} .
$$

The matrix of the linear system associated with the pressure equation involves the inverse of the mass matrix. In practice the presence of $\mathcal{I}_{h}^{-1}$ may hamper the practicability of the present approach in some circumstances. For instance, for finite element approximations the mass matrix is not diagonal, and the exact determination of the matrix of the pressure problem may become computationally very expensive, especially when a large number of unknowns is involved. In practice, a computationally convenient alternative approach consists in lumping the consistent mass matrix, by summing all elements of each row and placing the result on the diagonal (diagonal mass lumping), cf. Gresho and Chan [15, Part II] or Quartapelle [24, pp. 191-201]. Though this technique may work, no stability result has yet been proven.

It is the purpose of the next section to show that the mass matrix obstacle may be circumvented if the auxiliary space $Y_{h}$ in which the velocity is projected is chosen in an alternative different manner.

\subsection{The projection step as a Poisson problem}

We now choose $M_{h}$ as an internal approximation of $H^{1}(\Omega)$ (recall that in the previous sections we only required $M_{h} \subset L^{2}(\Omega)$ ). We also choose $Y_{h}=X_{h}+\nabla M_{h}$; note that this definition makes sense for $M_{h}$ being a subspace of $H^{1}(\Omega), \nabla M_{h}$ is in $L^{2}(\Omega)^{d}, Y_{h}$ is a subspace of $L^{2}(\Omega)^{d}$ as required by the theory developed above.

In this alternative framework the viscous step amounts to looking for $\tilde{u}_{h}^{k+1}$ in $X_{h}$ so that, for all $v_{h} \in X_{h}$

$$
\begin{aligned}
\frac{\left(\tilde{u}_{h}^{k+1}, v_{h}\right)-\left(u_{h}^{k}, v_{h}\right)}{\delta t}+a\left(\tilde{u}_{h}^{k+1}, v_{h}\right)+ & d\left(\tilde{u}_{h}^{k}, \tilde{u}_{h}^{k+1}, v_{h}\right) \\
& =\left(f^{k+1}, v_{h}\right)+\left(p_{h}^{k}, \nabla \cdot v_{h}\right) .
\end{aligned}
$$

Note that here $\tilde{u}_{h}^{k+1}$ and $u_{h}^{k}$ are approximated in different spaces. Furthermore, the projection step consists in finding $u_{h}^{k+1}$ in $Y_{h}$ and $p_{h}^{k+1}$ in $M_{h}$ so that

$$
\left\{\begin{array}{l}
\forall v_{h} \in Y_{h}, \quad\left(\frac{u_{h}^{k+1}-i_{h} \tilde{u}_{h}^{k+1}}{\delta t}, v_{h}\right)+\left(\nabla\left(p_{h}^{k+1}-p_{h}^{k}\right), v_{h}\right)=0 \\
\forall q_{h} \in M_{h}, \quad\left(u_{h}^{k+1}, \nabla q_{h}\right)=0 .
\end{array}\right.
$$

At first glance this formulation seems strange, even awkward, for $Y_{h}$ is not a classical space. Actually, the usefulness of $Y_{h}$ is emphasized by the following,

Numerische Mathematik Electronic Edition page 217 of Numer. Math. (1998) 80: 207-238 
Proposition 3.3. The projection step (3.11) is equivalent to the problem: Look for $p_{h}^{k+1}$ in $M_{h}$ so that

$$
\forall q_{h} \in M_{h}, \quad\left(\nabla\left(p_{h}^{k+1}-p_{h}^{k}\right), \nabla q_{h}\right)=-\frac{\left(\nabla \cdot \tilde{u}_{h}^{k+1}, q_{h}\right)}{\delta t},
$$

and set

$$
u_{h}^{k+1}=\tilde{u}_{h}^{k+1}-\delta t \nabla\left(p_{h}^{k+1}-p_{h}^{k}\right) .
$$

Proof. This is an easy consequence of Lemma 3.4 that follows.

Lemma 3.4. $C_{h}^{\mathrm{T}}$ is the restriction of $\nabla$ to $M_{h}$.

Proof. For all $\left(v_{h}, q_{h}\right)$ in $Y_{h} \times M_{h}$, we have $\left(C_{h}^{\mathrm{T}} q_{h}, v_{h}\right)=\left(\nabla q_{h}, v_{h}\right)$; that is to say $\left(C_{h}^{\mathrm{T}} q_{h}-\nabla q_{h}, v_{h}\right)=0$. But $\nabla q_{h}$ is in $Y_{h}$ by definition and $C_{h}^{\mathrm{T}} q_{h}$ is in $Y_{h}$; hence $C_{h}^{\mathrm{T}} q_{h}=\nabla q_{h}$.

In this case, the projection step amounts to solving a discrete Poisson problem for the pressure increment, $\left(p_{h}^{k+1}-p_{h}^{k}\right)$, supplemented with a homogeneous Neumann boundary condition on $\partial \Omega$. This type of discrete problem is very classical and the solution of the linear system associated with it has been subject to an enormous amount of research. Accurate and fast solvers for this problem are available.

Remark 3.5. Note that $u_{h}^{k+1}$ belongs to $X_{h}+\nabla M_{h}$ which is a subset of $L^{2}(\Omega)^{d}$. In general, the end-of-step velocity $u_{h}^{k+1}$ is discontinuous at inter element boundaries and thus its divergence has a meaning only as a distribution. For instance, if $P_{1}$ finite elements are used for approximating the pressure, the Laplacian of the pressure increment $\nabla^{2}\left(p_{h}^{k+1}-p_{h}^{k}\right)$ is a $H^{-1}(\Omega)$ measure, whereas $\nabla \cdot \tilde{u}_{h}^{k+1}$ is in $L^{2}(\Omega)$; hence, in this particular case, the divergence of $u_{h}^{k+1}$ is a $H^{-1}(\Omega)$ measure! However, it can be proved that $u_{h}^{k+1}$ converges weakly in $L^{2}(\Omega)^{d}$ to some divergence free vector field as the mesh is refined (i.e. the approximation parameter $h$ tends to zero).

Remark 3.6. As already mentioned above, the end-of-step velocity can be eliminated in practice. Hence the weird velocity space $Y_{h}$ is never used in practice.

\section{An extension with unconventional boundary conditions}

\subsection{Introduction}

We want now to illustrate the ability of the fractional-step projection method to accommodate unconventional boundary conditions. In particular we shall

Numerische Mathematik Electronic Edition page 218 of Numer. Math. (1998) 80: 207-238 
consider the imposition of derivative conditions on velocity and the enforcement of prescribed boundary values on pressure. We consider the following unsteady Navier-Stokes problem. For a given body force $f$ and a given divergence-free initial velocity field $u_{0}$, find a velocity field $u$ and a pressure field $p$ so that at $t=0, u=u_{0}$, and at all subsequent times

$$
\left\{\begin{array}{l}
\frac{\partial u}{\partial t}-\nabla^{2} u+(u \cdot \nabla) u+\nabla p=f, \\
\nabla \cdot u=0,
\end{array}\right.
$$

the velocity and the pressure being subject to the following boundary conditions:

$$
\begin{array}{rlrl}
u_{\mid \partial \Omega_{1}} & =0 ; & & \\
u \cdot n_{\mid \partial \Omega_{2}} & =0, & (\alpha n \times u+\nabla \times u) \times n_{\mid \partial \Omega_{2}}=0 ; \\
u \times n_{\mid \partial \Omega_{3}} & =0, & p_{\mid \partial \Omega_{3}}=0 ;
\end{array}
$$

where $\partial \Omega_{1}, \partial \Omega_{2}, \partial \Omega_{3}$ is a partition of $\partial \Omega$. The function $\alpha$ is defined on $\partial \Omega_{2}$ and is assumed to be suitably smooth.

\subsection{The variational formulation}

To recast problem (4.1)-(4.2) in a variational form, we introduce the following Hilbert spaces:

$$
\begin{gathered}
V=\{v \in X, \nabla \cdot v=0\} \\
H=\left\{v \in L^{2}(\Omega)^{d}, \nabla \cdot v=0, v \cdot n_{\mid \partial \Omega_{1} \cup \partial \Omega_{2}}=0\right\}
\end{gathered}
$$

Denote by $H_{0, \partial \Omega_{3}}^{1}(\Omega)$ the space of scalar functions of $H^{1}(\Omega)$ the trace of which is zero on $\partial \Omega_{3}$. The importance of $H$ and $H_{0, \partial \Omega_{3}}^{1}(\Omega)$ is emphasized by the orthogonal decomposition of $L^{2}(\Omega)^{d}$.

Proposition 4.1. $L^{2}(\Omega)^{d}=H \oplus \nabla\left(H_{0, \partial \Omega_{3}}^{1}(\Omega)\right)$.

Proof. For all $f$ in $L^{2}(\Omega)^{d}$, denote by $p$ the unique solution in $H_{0, \partial \Omega_{3}}^{1}(\Omega)$ of the following problem

$$
\forall q \in H_{0, \partial \Omega_{3}}^{1}(\Omega), \quad(\nabla p, \nabla q)=(f, \nabla q) .
$$

It is an easy matter to verify that $v=f-\nabla p$ is in $H$.

Numerische Mathematik Electronic Edition page 219 of Numer. Math. (1998) 80: 207-238 
This decomposition (actually its discrete counterpart) is the one playing a key role in the projection technique under the unconventional boundary conditions (4.2). For the sake of simplicity, it is hereafter assumed that meas $\left(\partial \Omega_{3}\right)>0$ so that the pressure is uniquely defined in $M$. If this hypothesis is not satisfied, a unique pressure is selected by setting $M=$ $L_{0}^{2}(\Omega)$ (see also the proof of Lemma 4.2).

To simplify the notations and put problem (4.1) in a variational framework, we define the linear continuous operators $A_{1}$ and $A: X \longrightarrow X^{\prime}$ so that for all $v \in X$,

$$
\left\langle A_{1} u, v\right\rangle=(\nabla \cdot u, \nabla \cdot v)+(\nabla \times u, \nabla \times v)
$$

and

$$
\langle A u, v\rangle=\left\langle A_{1} u, v\right\rangle+\int_{\partial \Omega_{2}} \alpha(u \times n) \cdot(v \times n) .
$$

As in Sect. 2, we introduce the operator $B: X \longrightarrow M$ so that for all $v \in X$ and $q \in M$,

$$
(B v, q)=-(\nabla \cdot v, q)
$$

Concerning $B$, we can prove

Lemma 4.2. $B$ is surjective.

The demonstration of this result is quite classical when Dirichlet conditions are enforced on velocity on the entire boundary, that is when $X=H_{0}^{1}(\Omega)^{d}$. The result is less known in the general case we are considering, hence we reproduce a proof kindly suggested to the authors by Vivette Girault.

Proof. Assuming that there is a little patch $\mathcal{O}$ of $\partial \Omega_{3}$ where $\partial \Omega_{3}$ is smooth, for example if $\partial \Omega_{3}$ is a polyhedron, Lemma 4.2 can be proved constructively. Let $q$ be given in $M$, fix a smooth non negative function $\rho$ with compact support on $\mathcal{O}$ and define $g=c \rho n$ on $\mathcal{O}$, where $c$ is a scalar constant chosen such that $\int_{\mathcal{O}} g \cdot n=\int_{\Omega} q$. Then extend $g$ by zero to the whole boundary $\partial \Omega$. As $\mathcal{O}$ is smooth, $g \in H^{1 / 2}(\partial \Omega)^{d}$; besides $g \times n=0$ on $\partial \Omega_{3}$ and $g=0$ elsewhere. Lift $g$ by a function $w$ in $H^{1}(\Omega)^{d}$; clearly $w \in X$. Set $q_{0}=\nabla \cdot w-q$; then $q_{0} \in L_{0}^{2}(\Omega)$ and, given the surjectivity of the mapping $\nabla \cdot: H_{0}^{1}(\Omega)^{d} \longrightarrow L_{0}^{2}(\Omega)$ (cf. Girault-Raviart [14, p. 24]), there exists $w_{0} \in H_{0}^{1}(\Omega)^{d}$ such that $\nabla \cdot w_{0}=q_{0}$. Thus $w-w_{0}$ is the required function.

Concerning $A_{1}$, we can prove

Lemma 4.3. Provided $\partial \Omega$ is smooth enough and meas $\left(\partial \Omega_{1}\right)>0, A_{1}$ is $X$-elliptic in the sense that there is $c>0$ so that for all $v$ in $X,\left\langle A_{1} v, v\right\rangle \geq$ $c\|v\|_{1}^{2}$.

Numerische Mathematik Electronic Edition page 220 of Numer. Math. (1998) 80: 207-238 
Proof. The demonstration of this result is not very well-known when boundary conditions are mixed, so we give a proof (without claiming originality).

(i) First we prove that $A_{1}$ is injective. If $\Omega$ is not simply connected, i.e. $\Omega$ is $p$-connected, we define $p$ cuts $\Sigma_{1}, \ldots, \Sigma_{p}$ so that the cuts in question are smooth manifolds of dimension $d-1, \Sigma_{i} \cap \Sigma_{j}=\emptyset$ if $i \neq j$, and $\dot{\Omega}=\Omega \backslash \bigcup_{i=1}^{p} \Sigma_{i}$ is simply connected and smooth. Let $u$ be in $X$ and assume that $A_{1} u=0$, then $\nabla \cdot u=0$ and $\nabla \times u=0$ in $\dot{\Omega}$. Given the simple connectedness of $\dot{\Omega}$, this means that $u$ is the gradient of a harmonic scalar function $\phi$. The boundary condition $u_{\mid \partial \Omega_{1}}=0$ means that $(\partial \phi / \partial n)_{\mid \partial \Omega_{1}}=0$ and $\phi_{\mid \partial \Omega_{1}}=$ constant. Given the hypothesis meas $\left(\partial \Omega_{1}\right)>0$ and the extension theorem of harmonic functions (cf. e.g. Dautray and Lions [12, Chap. II, p. 308]) we infer that $\phi$ is a constant in $\dot{\Omega}$; as a result, $\phi$ is constant almost everywhere in $\Omega$. That is to say $u$ is zero almost everywhere in $\Omega$; hence, the class representative of $u$ in $X$ (in the sense of the Lebesgue measure) is zero.

(ii) Let $u$ and $v$ be some smooth functions in $X$. Irrespective of any boundary condition assumed by $u$ and $v$, an integration by parts yields

$$
(\nabla u, \nabla v)=\left\langle A_{1} u, v\right\rangle+\int_{\partial \Omega}\left[(\nabla \times u) \cdot v \times n-(\nabla \cdot u) v \cdot n+\frac{\partial u}{\partial n} \cdot v\right] .
$$

Denote by $I_{\partial \Omega}(u, v)$ the boundary integral in the right-hand side. Provided $\partial \Omega$ is smooth enough, given the boundary conditions enforced on the functions of $X$ (namely $w_{\mid \partial \Omega_{1}}=0, w \cdot n_{\mid \partial \Omega_{2}}=0, w \times n_{\mid \partial \Omega_{3}}=0$ ) the surface integral can be bounded (after some calculus) as follows

$$
I_{\partial \Omega}(u, v) \leq c \int_{\partial \Omega}|u \cdot v|
$$

For other details on the way of obtaining this inequality, the reader is referred to Dautray and Lions [12, Chap. IX, p. 246]. From the Poincaré inequality and the inequality above, we infer the result

$$
c\|u\|_{1}^{2} \leq\left\langle A_{1} u, u\right\rangle+c^{\prime} \int_{\partial \Omega}|u|^{2} .
$$

(iii) Thanks to (i), (ii) and the fact that the embedding of $H^{1 / 2}(\partial \Omega)$ into $L^{2}(\partial \Omega)$ is compact, Peetre-Tartar's Lemma yields the desired inequality

$$
c\|u\|_{1}^{2} \leq\left\langle A_{1} u, u\right\rangle .
$$

Hence $A_{1}$ is elliptic on $X$.

Remark 4.1. The hypothesis meas $\left(\partial \Omega_{1}\right)>0$ plays a key role in the proof of the injectivity of $A_{1}$. For other details on this matter the reader is referred to Girault and Raviart [14, p. 51-56].

Numerische Mathematik Electronic Edition page 221 of Numer. Math. (1998) 80: 207-238 
We now consider the following variational problem. For $f \in$ $W^{2, \infty}\left(0, \infty ; L^{2}(\Omega)^{d}\right)$ and $u_{0} \in V \cap H^{2}(\Omega)^{d}$, find a pair $(u, p)$

$$
\begin{aligned}
& u \in L^{\infty}(0, T ; H) \cap L^{2}(0, T ; V), u_{t} \in L^{2}\left(0, T ; H^{-1}\right), \quad \text { for all } T>0, \\
& p \in L^{2}(0, T ; M), \quad \text { for all } T>0,
\end{aligned}
$$

and

$$
\left\{\begin{array}{l}
u_{t}+A u+D(u, u)+B^{\mathrm{T}} p=f, \\
B u=0 \\
u(0)=u_{0} .
\end{array}\right.
$$

Remark 4.2. The conditions on the trace, the normal trace and the tangential trace of the velocity on $\partial \Omega_{1}, \partial \Omega_{2}$ and $\partial \Omega_{3}$, respectively, are all essential boundary conditions; they are enforced by the definition (4.3) of the Hilbert space $X$. Note that these boundary conditions are understood in some weak sense, the exact meaning of which is out of the scope of the present paper, cf. e.g. Lions and Magenes [23]. The condition involving the tangential components of $\nabla \times u$ on $\partial \Omega_{2}$ is a natural boundary condition of Robin type. The pressure boundary condition on $\partial \Omega_{3}$ is natural as well. Actually, the weak formulation enforces the natural boundary condition $(\nabla \cdot u-p)_{\mid \partial \Omega_{3}}=$ 0 .

Remark 4.3. The reader can verify that the present variational formulation could also enforce the natural boundary conditions $p_{\mid \partial \Omega_{4}}=0$ and $(\alpha n \times$ $u+\nabla \times u) \times n_{\mid \partial \Omega_{4}}=0$. Actually, these conditions are not recommended for it can be shown that, though $A_{1}$ remains injective, $A_{1}$ is no longer $X$-elliptic (Bernardi-Girault-Sanchez-Palencia [5], and Guermond-Quartapelle [20]). It seems important for this formulation to be stable that on every piece of the boundary (at least one of) either the normal or the tangential component of the velocity is prescribed.

Remark 4.4. Note that the use of the bilinear form $(\nabla \cdot u, \nabla \cdot v)+(\nabla \times u, \nabla \times v)$ is mandatory when the boundaries $\partial \Omega_{2}$ and $\partial \Omega_{3}$, where the prescribed boundary conditions are different from a purely Dirichlet condition for the velocity alone, are curved. On the contrary, the more common bilinear form $(\nabla u, \nabla v)$ can be used when Dirichlet conditions are specified or when the boundaries $\partial \Omega_{2}$ and $\partial \Omega_{3}$ are flat and parallel to the Cartesian axes. Note also that the coupling between the velocity components, generated by the presence of mixed boundary conditions, appears in the definition of the test functions of $X$.

A complete analysis of (4.9) is out of the scope of the present paper; we assume that the problem is well-posed and that the solution has the required smoothness if the data are smooth enough. We denote by $X_{h}$ and $M_{h}$ conformal approximations of $X$ and $M$. In the following we consider the abstract time-dependent problem (2.15) within the present functional

Numerische Mathematik Electronic Edition page 222 of Numer. Math. (1998) 80: 207-238 
framework, and we are interested in approximating this problem by means of a projection algorithm for $t \geq t_{0} \geq 0$.

Remark 4.5. Note that the conservative form of the nonlinear term described above is frequently used in textbooks along with the hypothesis meas $\left(\partial \Omega_{3}\right)=0$. Without pretending the originality we point out the fact that this form can also be used for channel flows with an "open" boundary, provided the "open" boundary in question is located downstream, far enough of any recirculatory zone, so that the condition $u_{h} \cdot n_{\mid \partial \Omega_{3}} \geq 0$ is guaranteed, since in this case we have

Proposition 4.4. Let $u_{h}$ be in $X_{h}$. Provided either meas $\left(\partial \Omega_{3}\right)=0$ or $u_{h} \cdot n_{\mid \partial \Omega_{3}} \geq 0$, for all $v_{h}$ in $X_{h}$ the following inequality holds

$$
\left(D_{h}\left(u_{h}, v_{h}\right), v_{h}\right) \geq 0 \text {. }
$$

Proof. Let $\left(u_{h}, v_{h}, w_{h}\right) \in\left(X_{h}\right)^{3}$; by integration by parts we have

$$
\int_{\Omega}\left[\left(u_{h} \cdot \nabla\right) v_{h}\right] \cdot w_{h}=-\int_{\Omega}\left[\left(u_{h} \cdot \nabla\right) w_{h}\right] \cdot v_{h}-\left(\nabla \cdot u_{h}, v_{h} \cdot w_{h}\right)+\int_{\partial \Omega_{3}} u_{h} \cdot n\left(v_{h} \cdot w_{h}\right) .
$$

This implies

$$
\left(D_{h}\left(u_{h}, v_{h}\right), v_{h}\right)=\frac{1}{2} \int_{\partial \Omega_{3}} u_{h} \cdot n\left|v_{h}\right|^{2}
$$

which, given the hypotheses on either $u_{h} \cdot n_{\mid \partial \Omega_{3}}$ or $\partial \Omega_{3}$, yields the desired result.

In practice, this treatment of the nonlinear term can guarantee some "unconditional" stability to the numerical scheme (see [19] for numerical tests).

\subsection{The projection step as a Darcy problem}

Theoretically it is not necessary to consider nonhomogeneous boundary values of pressure on $\partial \Omega_{3}$, for some smooth lifting of the pressure can be invoked so that the new pressure satisfies a homogeneous boundary condition. This theoretical argument is perfectly correct; however, the practical application of this principle may not be obvious, so we give some details. We assume in this section and in the following that the pressure is prescribed to satisfy a nonhomogeneous boundary condition on $\partial \Omega_{3}$, namely,

$$
p_{\mid \partial \Omega_{3}}=P
$$

where the function $P$ is defined only on $\partial \Omega_{3}$ and may depend on time in general. The definition of the functional framework remains unchanged. The

Numerische Mathematik Electronic Edition page 223 of Numer. Math. (1998) 80: 207-238 
only thing that changes is the right-hand side of the momentum equation in variational form (4.9), which should be replaced by

$$
(f, v)-\int_{\partial \Omega_{3}} P v \cdot n .
$$

As a consequence, the discrete problem (2.15) is modified only in the righthand side of the discrete momentum equation. Namely, the linear form $f_{h}$ must be replaced by $g_{h}: X_{h} \longrightarrow X_{h}$ so that, for all $v_{h} \in X_{h},\left(g_{h}, v_{h}\right)=$ $\left(f, v_{h}\right)-\int_{\partial \Omega_{3}} P v_{h} \cdot n$. The projection algorithm as described above remains unchanged provided $f_{h}^{k+1}$ is replaced by $g_{h}^{k+1}$ in the viscous step (3.3), where $\left(g_{h}^{k+1}, v_{h}\right)=\left(f^{k+1}, v_{h}\right)-\int_{\partial \Omega_{3}} P^{k+1} v_{h} \cdot n$.

If we choose $Y_{h}=X_{h}$ the viscous step consists of looking for $\tilde{u}_{h}^{k+1}$ in $X_{h}$ so that, for all $v_{h} \in X_{h}$

$$
\begin{aligned}
\left(\frac{\tilde{u}_{h}^{k+1}-u_{h}^{k}}{\delta t}, v_{h}\right)+ & a\left(\tilde{u}_{h}^{k+1}, v_{h}\right)+d\left(\tilde{u}_{h}^{k}, \tilde{u}_{h}^{k+1}, v_{h}\right) \\
= & \left(f^{k+1}, v_{h}\right)+\left(p_{h}^{k}, \nabla \cdot v_{h}\right)-\int_{\partial \Omega_{3}} P^{k+1} v_{h} \cdot n .
\end{aligned}
$$

Since no distinction needs to be made between $C_{h}$ and $B_{h}$, the projection step reads: find $u_{h}^{k+1}$ in $X_{h}$ and $p_{h}^{k+1}$ in $M_{h}$ so that

$$
\begin{cases}\forall v_{h} \in X_{h}, & \left(\frac{u_{h}^{k+1}-\tilde{u}_{h}^{k+1}}{\delta t}, v_{h}\right)-\left(p_{h}^{k+1}-p_{h}^{k}, \nabla \cdot v_{h}\right)=0 \\ \forall q_{h} \in M_{h}, & \left(\nabla \cdot u_{h}^{k+1}, q_{h}\right)=0\end{cases}
$$

Remark 4.6. The discrete viscous step enforces weakly the nonhomogeneous natural boundary condition $\nabla \cdot \tilde{u}_{h}^{k+1} \mid \partial \Omega_{3}=p_{h \mid \partial \Omega_{3}}^{k}-P^{k+1}$.

Remark 4.7. In the projection step no essential boundary condition on the pressure is enforced; though in the weak sense the projection step naturally enforces the (always) homogeneous boundary condition $\left(p_{h}^{k+1}-p_{h}^{k}\right)_{\mid \partial \Omega_{3}}=$ 0 .

\subsection{The projection step as a Poisson problem}

We now choose $M_{h}$ as an internal approximation of $H^{1}(\Omega)$; however, for we want $C_{h}$ to be an extension of $B_{h}$ we are led to set $M_{h} \subset H_{0, \partial \Omega_{3}}^{1}(\Omega)$ where

$$
H_{0, \partial \Omega_{3}}^{1}(\Omega)=\left\{q \in H^{1}(\Omega), q_{\mid \partial \Omega_{3}}=0\right\} .
$$

Numerische Mathematik Electronic Edition page 224 of Numer. Math. (1998) 80: 207-238 
We also choose $Y_{h}=X_{h}+\nabla M_{h}$. In this alternative framework the viscous step amounts to looking for $\tilde{u}_{h}^{k+1}$ in $X_{h}$ so that, for all $v_{h} \in X_{h}$

$$
\begin{gathered}
\frac{\left(\tilde{u}_{h}^{k+1}, v_{h}\right)-\left(u_{h}^{k}, v_{h}\right)}{\delta t}+a\left(\tilde{u}_{h}^{k+1}, v_{h}\right)+d\left(\tilde{u}_{h}^{k}, \tilde{u}_{h}^{k+1}, v_{h}\right) \\
=\left(f^{k+1}, v_{h}\right)-\left(\nabla p_{h}^{k}, v_{h}\right) .
\end{gathered}
$$

Given the choice $M_{h} \subset H_{0, \partial \Omega_{3}}^{1}(\Omega)$, we can prove that $C_{h}^{\mathrm{T}}$ is the restriction of $\nabla$ to $M_{h}$; as a result, the projection step reads: Find $p_{h}^{k+1}$ in $M_{h}$ so that $p_{h}^{k+1}{ }_{\mid \partial \Omega_{3}}=P^{k+1}$ and

$$
\forall q_{h} \in M_{h}, \quad\left(\nabla\left(p_{h}^{k+1}-p_{h}^{k}\right), \nabla q_{h}\right)=-\frac{\left(\nabla \cdot \tilde{u}_{h}^{k+1}, q_{h}\right)}{\delta t},
$$

and set

$$
u_{h}^{k+1}=\tilde{u}_{h}^{k+1}-\delta t \nabla\left(p_{h}^{k+1}-p_{h}^{k}\right) .
$$

Remark 4.8. The projection step amounts to solving a discrete Poisson problem supplemented with a homogeneous natural Neumann boundary condition on $\partial \Omega_{1} \cup \partial \Omega_{2}$ and an essential Dirichlet boundary condition on $\partial \Omega_{3}$.

\section{The error analysis}

We now turn our attention to the error analysis of the projection scheme but we restrict ourselves to homogeneous Dirichlet conditions on the velocity. Actually, all that is said below would apply also to the case of the unconventional boundary conditions discussed so far, provided we had at hand regularity estimates and assuming that $u(t) \cdot n_{\mid \partial \Omega_{3}} \geq 0$, for $t>0$.

\subsection{Preliminaries}

Before going through the details of the error analysis, we introduce some technical tools.

For a Banach space $W$, we denote by $L^{p}(W)$ the space $L^{p}\left(t^{k_{0}}, T ; W\right)$. We also denote by $l^{p}(W)$ the space $\left\{\left(w^{k_{0}}, \ldots, w^{K}\right) ; w^{k} \in W, k_{0} \leq k \leq\right.$ $K$ \} equipped with the norm

$$
\begin{aligned}
\|w\|_{l^{p}(W)} & =\left(\delta t \sum_{k=k_{0}}^{K}\left\|w^{k}\right\|_{W}^{p}\right)^{1 / p}, \quad \text { for } 1 \leq p<\infty, \\
\|w\|_{l^{\infty}(W)} & =\max _{k_{0} \leq k \leq K}\left\|w^{k}\right\|_{W} .
\end{aligned}
$$

We define interpolates of $u(t)$ and $p(t)$ that preserve the high approximation order $h^{l+1}$ on $u(t)$ in the $L^{2}(\Omega)^{d}$-norm without having to rely on

Numerische Mathematik Electronic Edition page 225 of Numer. Math. (1998) 80: 207-238 
duality arguments. For all $t$, we define $\left(w_{h}(t), q(t)\right) \in X_{h} \times M_{h}$ as the solution of the following discrete Stokes problem:

(5.2) $\left\{\begin{array}{c}\left(\nabla w_{h}(t), \nabla v_{h}\right)+\left(B_{h}^{\mathrm{T}} q_{h}(t), v_{h}\right)=\left(\nabla u(t), \nabla v_{h}\right)-\left(p(t), \nabla \cdot v_{h}\right), \\ \forall v_{h} \in X_{h}, \\ \left(B_{h} w_{h}(t), r_{h}\right)=-\left(\nabla \cdot u(t), r_{h}\right), \\ \forall r_{h} \in M_{h} .\end{array}\right.$

Given the $H^{2}$-regularity of the Stokes operator in regular domains together with a classical duality argument, these interpolates can be shown to satisfy

Lemma 5.1. Provided $u^{(j)} \in L^{\beta}\left(H^{l+1}(\Omega)^{d} \cap V\right), p^{(j)} \in L^{\beta}\left(H^{l}(\Omega) \cap M\right)$ for $1 \leq \beta \leq \infty$, there exists $c>0$ so that

$$
\begin{aligned}
& \left\|u^{(j)}-w_{h}^{(j)}\right\|_{L^{\beta}\left(L^{2}(\Omega)^{d}\right)} \\
& +h\left[\left\|u^{(j)}-w_{h}^{(j)}\right\|_{L^{\beta}\left(H^{1}(\Omega)^{d}\right)}+\left\|p^{(j)}-q_{h}^{(j)}\right\|_{L^{\beta}\left(L^{2}(\Omega)\right)}\right] \\
& \quad \leq c h^{l+1}\left[\left\|u^{(j)}\right\|_{L^{\beta}\left(H^{l+1}(\Omega)^{d}\right)}+\left\|p^{(j)}\right\|_{L^{\beta}\left(H^{l}(\Omega)\right)}\right] .
\end{aligned}
$$

Lemma 5.2. Provided $u^{(j)} \in L^{\beta}\left(H^{2}(\Omega)^{d} \cap X\right), p^{(j)} \in L^{\beta}\left(H^{1}(\Omega) \cap M\right)$, for $1 \leq \beta \leq \infty$, then there exists $c>0$ such that

$$
\begin{aligned}
& \left\|w_{h}^{(j)}\right\|_{L^{\beta}\left(W^{0, \infty}(\Omega)^{d} \cap W^{1,3}(\Omega)^{d}\right)} \\
& \quad \leq c\left(\left\|u^{(j)}\right\|_{L^{\beta}\left(H^{2}(\Omega)^{d}\right)}+\left\|p^{(j)}\right\|_{L^{\beta}\left(H^{1}(\Omega)\right)}\right) .
\end{aligned}
$$

Furthermore, the interpolate $q_{h}^{(j)}$ satisfies the following stability result:

Lemma 5.3. Provided $p^{(j)}$ is in $L^{\beta}\left(H^{1}(\Omega) \cap M\right)$ and $u^{(j)} \in L^{\beta}\left(H^{2}(\Omega)^{d} \cap\right.$ $\left.H_{0}^{1}(\Omega)^{d}\right)$ for $1 \leq \beta \leq \infty$, we have

$$
\left\|C_{h}^{\mathrm{T}} q_{h}^{(j)}\right\|_{L^{\beta}\left(L^{2}(\Omega)^{d}\right)} \leq c\left(\left\|p^{(j)}\right\|_{L^{\beta}\left(H^{1}(\Omega)\right)}+\left\|u^{(j)}\right\|_{L^{\beta}\left(H^{2}(\Omega)^{d}\right)}\right) .
$$

Proof. (i) Assume $Y_{h} \subset H_{0}^{\operatorname{div}}(\Omega)$. Using an inverse inequality and the approximation property of $q_{h}^{(j)}$, we deduce

$$
\begin{aligned}
\left\|C_{h}^{\mathrm{T}} q_{h}^{(j)}\right\|_{0}^{2} & =\left(C_{h}^{\mathrm{T}} q_{h}^{(j)}, C_{h}^{\mathrm{T}} q_{h}^{(j)}\right) \\
& =-\left(\nabla \cdot\left(C_{h}^{\mathrm{T}} q_{h}^{(j)}\right), q_{h}^{(j)}\right) \\
& =-\left(\nabla \cdot\left(C_{h}^{\mathrm{T}} q_{h}^{(j)}\right), q_{h}^{(j)}-p^{(j)}\right)-\left(\nabla \cdot\left(C_{h}^{\mathrm{T}} q_{h}^{(j)}\right), p^{(j)}\right) \\
& \leq c h^{-1}\left\|C_{h}^{\mathrm{T}} q_{h}^{(j)}\right\|_{0}\left\|q_{h}^{(j)}-p^{(j)}\right\|_{0}+\left(C_{h}^{\mathrm{T}} q_{h}^{(j)}, \nabla p^{(j)}\right) \\
& \leq c\left\|C_{h}^{\mathrm{T}} q_{h}^{(j)}\right\|_{0}\left(\left\|u^{(j)}\right\|_{2}+\left\|p^{(j)}\right\|_{1}\right),
\end{aligned}
$$

that is to say,

$$
\left\|C_{h}^{\mathrm{T}} q_{h}^{(j)}\right\|_{0} \leq c\left(\left\|u^{(j)}\right\|_{2}+\left\|p^{(j)}\right\|_{1}\right) .
$$

Numerische Mathematik Electronic Edition page 226 of Numer. Math. (1998) 80: 207-238 
(ii) Assume $M_{h} \subset H^{1}(\Omega)$

$$
\begin{aligned}
\left\|C_{h}^{\mathrm{T}} q_{h}^{(j)}\right\|_{0}^{2} & =\left(\nabla q_{h}^{(j)}, C_{h}^{\mathrm{T}} q_{h}^{(j)}\right) \\
& \leq\left\|q_{h}^{(j)}\right\|_{1}\left\|C_{h}^{\mathrm{T}} q_{h}^{(j)}\right\|_{0} \\
& \leq c\left(\left\|u^{(j)}\right\|_{2}+\left\|p^{(j)}\right\|_{1}\right)\left\|C_{h}^{\mathrm{T}} q_{h}^{(j)}\right\|_{0} .
\end{aligned}
$$

This completes the proof of the lemma.

We shall use repeatedly a discrete version of the Gronwall inequality. For the sake of completeness we recall the result but for the sake of shortness we refer to Heywood and Rannacher [21, IV, p. 369] for a proof, or to Quarteroni and Valli [25, p. 14] for a simplified version of it.

Lemma 5.4. Let $\delta, g_{0}, a_{n}, b_{n}, c_{n}$ and $\gamma_{n}$ be a sequence of non negative numbers for integers $n \geq 0$ so that

$$
a_{n}+\delta \sum_{j=0}^{n} b_{j} \leq \delta \sum_{j=0}^{n} \gamma_{j} a_{j}+\delta \sum_{j=0}^{n} c_{j}+g_{0} .
$$

Assume that $\gamma_{j} \delta<1$ for all $j$, and set $\sigma_{j}=\left(1-\gamma_{j} \delta\right)^{-1}$. Then, for all $n \geq 0$,

$$
a_{n}+\delta \sum_{j=0}^{n} b_{j} \leq \exp \left(\delta \sum_{j=0}^{n} \sigma_{j} \gamma_{j}\right)\left[\delta \sum_{j=0}^{n} c_{j}+g_{0}\right]
$$

\subsection{Error bounds}

For the sake of conciseness we set

$$
e_{h}^{k}=w_{h}\left(t^{k}\right)-u_{h}^{k}, \quad \tilde{e}_{h}^{k}=w_{h}\left(t^{k}\right)-\tilde{u}_{h}^{k}, \quad \epsilon_{h}^{k}=q_{h}\left(t^{k}\right)-p_{h}^{k},
$$

where $(u(t), p(t))$ is the solution of the continuous problem (2.10). For simplicity we assume that the projection algorithm is initialized so that

$$
\begin{gathered}
\left\|e_{h}^{k_{0}}\right\|_{0} \leq c\left(\delta t+h^{l+1}\right), \\
\left\|\tilde{e}_{h}^{k_{0}}\right\|_{1} \leq c\left(\delta t+h^{l+1}\right) / \delta t^{1 / 2}, \\
\left\|C_{h}^{\mathrm{T}} \epsilon_{h}^{k_{0}}\right\|_{0} \leq c .
\end{gathered}
$$

Under this hypothesis and provided the solution of (2.10) is such that $u, u_{t}$ are in $L^{\infty}\left(H^{l+1}(\Omega)^{d}\right), u_{t t} \in L^{\infty}\left(H^{1}(\Omega)^{d}\right)$, and $p, p_{t}$ are in $L^{\infty}\left(H^{l}(\Omega)\right), p_{t t} \in$ $L^{\infty}\left(L^{2}(\Omega)\right)$ then the ability of the solution of (3.3)-(3.4) to approximate that of (2.10) for $k_{0} \leq k \leq K$ is given by

Numerische Mathematik Electronic Edition page 227 of Numer. Math. (1998) 80: 207-238 
Theorem 5.5. Provided $\delta t$ is small enough, the solution to the projection scheme (3.3)-(3.4) satisfies:

$$
\begin{gathered}
\left\|u-u_{h}\right\|_{l^{\infty}\left(L^{2}(\Omega)^{d}\right)}+\left\|u-\tilde{u}_{h}\right\|_{l^{\infty}\left(L^{2}(\Omega)^{d}\right)} \leq c\left(h^{l+1}+\delta t\right), \\
\left\|u-\tilde{u}_{h}\right\|_{l^{2}\left(H^{1}(\Omega)^{d}\right)} \leq c\left(h^{l}+\delta t\right) .
\end{gathered}
$$

Proof. (a) For conciseness we denote $u^{k}=u\left(t^{k}\right)$ and $p^{k}=p\left(t^{k}\right)$, and for any function of time, $\phi(t)$, we introduce the notation $\delta_{t} \phi^{k+1}=\phi^{k+1}-\phi^{k}$. Given the particular interpolate $\left(w_{h}, q_{h}\right)$ that we have chosen, the solution to the Navier-Stokes problem (2.10) satisfies at time $t^{k+1}$

$$
\left\{\begin{array}{l}
\frac{\delta_{t} w_{h}^{k+1}}{\delta t}+A_{h} w_{h}^{k+1}+B_{h}^{\mathrm{T}} q_{h}^{k+1} \\
\quad=i_{X_{h}}^{\mathrm{T}}\left[f^{k+1}+R^{k+1}-D\left(u^{k+1}, u^{k+1}\right)\right], \\
B_{h} w_{h}^{k+1}=0,
\end{array}\right.
$$

where we have set

$$
R^{k+1}=\frac{\delta_{t} w_{h}^{k+1}}{\delta t}-u_{t}^{k+1}=w_{h, t}^{k+1}-u_{t}^{k+1}-\frac{1}{\delta t} \int_{t^{k}}^{t^{k+1}}\left(s-t^{k}\right) w_{h, s s} d s .
$$

By subtracting the first equation of (3.3) from (5.11), we derive the equation that controls the error $\tilde{e}_{h}^{k+1}$

$$
\frac{\tilde{e}_{h}^{k+1}-i_{h}^{\mathrm{T}} e_{h}^{k}}{\delta t}+A_{h} \tilde{e}_{h}^{k+1}+B_{h}^{\mathrm{T}} \psi_{h}^{k}=i_{X_{h}}^{\mathrm{T}}\left[R^{k+1}+R_{\mathrm{n} .1 .}^{k+1}\right]
$$

where we have set

$$
R_{\mathrm{n} .1 .}^{k+1}=-D\left(u^{k+1}, u^{k+1}\right)+D_{h}\left(\tilde{u}_{h}^{k}, \tilde{u}_{h}^{k+1}\right),
$$

and

$$
\psi_{h}^{k}=q_{h}^{k+1}-p_{h}^{k}=\delta_{t} q_{h}^{k+1}+\epsilon_{h}^{k} .
$$

Furthermore, using the fact that $w_{h}^{k+1} \in X_{h}, B_{h} w_{h}^{k+1}=0$, and $C_{h}$ is an extension of $B_{h}$, we obtain the system of equations that controls $e_{h}^{k+1}$ and $\epsilon_{h}^{k+1}$

$$
\left\{\begin{array}{l}
\frac{e_{h}^{k+1}-i_{h} \tilde{e}_{h}^{k+1}}{\delta t}+C_{h}^{\mathrm{T}}\left(\epsilon_{h}^{k+1}-\psi_{h}^{k}\right)=0, \\
C_{h} e_{h}^{k+1}=0 .
\end{array}\right.
$$

(b) To obtain a bound on $\tilde{e}_{h}^{k+1}$, we take the inner product of (5.12) by $2 \delta t \tilde{e}_{h}^{k+1}$. Using the $X$-ellipticity of $A_{h}$ (the ellipticity constant is hereafter denoted by $\alpha$ ) together with the classical relation $2(a, a-b)=\|a\|^{2}+\| a-$ $b\left\|^{2}-\right\| b \|^{2}$ we obtain:

$$
\begin{aligned}
\left\|\tilde{e}_{h}^{k+1}\right\|_{0}^{2}+\left\|\tilde{e}_{h}^{k+1}-{ }_{h}^{\mathrm{T}} e_{h}^{k}\right\|_{0}^{2}-\left\|i_{h}^{\mathrm{T}} e_{h}^{k}\right\|_{0}^{2}+2 \alpha \delta t\left\|\tilde{e}_{h}^{k+1}\right\|_{1}^{2} \\
+2 \delta t\left(\tilde{e}_{h}^{k+1}, B_{h}^{\mathrm{T}} \psi_{h}^{k}\right) \leq 2 \delta t\left\langle R^{k+1}+R_{\text {n. l. }}^{k+1}, \tilde{e}_{h}^{k+1}\right\rangle .
\end{aligned}
$$

Numerische Mathematik Electronic Edition page 228 of Numer. Math. (1998) 80: 207-238 
Using the classical inequality $2 a b \leq \gamma\|a\|^{2}+\|b\|^{2} / \gamma$ for all $\gamma>0$ together with the regularity of $u$ and $p$, the first term in the right-hand side is bounded from above as follows

$$
\begin{aligned}
2 \delta t\left\langle R^{k+1}, \tilde{e}_{h}^{k+1}\right\rangle & \leq 2 \delta t\left\|R^{k+1}\right\|_{-1}\left\|\tilde{e}_{h}^{k+1}\right\|_{1} \\
& \leq \gamma \delta t\left\|\tilde{e}_{h}^{k+1}\right\|_{1}^{2}+c_{\gamma} \delta t\left\|R^{k+1}\right\|_{-1}^{2} \\
& \leq \gamma \delta t\left\|\tilde{e}_{h}^{k+1}\right\|_{1}^{2}+c_{\gamma} \delta t\left(h^{l+1}+\delta t\right)^{2} .
\end{aligned}
$$

In the following, $c_{\gamma}$ is a generic constant that depends on the generic constant $\gamma$ which can be chosen as small as needed.

Now, we control the residual involving the nonlinear terms. Note first that we have

$$
\begin{aligned}
-\left\langle R_{\mathrm{n} .1 .}^{k+1}, \tilde{e}_{h}^{k+1}\right\rangle= & d\left(\delta_{t} u^{k+1}, u^{k+1}, \tilde{e}_{h}^{k+1}\right)+d\left(u^{k}-w_{h}^{k}, u^{k+1}, \tilde{e}_{h}^{k+1}\right) \\
& +d\left(w_{h}^{k}, u^{k+1}-w_{h}^{k+1}, \tilde{e}_{h}^{k+1}\right)+d\left(w_{h}^{k}-\tilde{u}_{h}^{k}, w_{h}^{k+1}, \tilde{e}_{h}^{k+1}\right) \\
& +d\left(\tilde{u}_{h}^{k}, w_{h}^{k+1}-\tilde{u}_{h}^{k+1}, \tilde{e}_{h}^{k+1}\right) .
\end{aligned}
$$

We now give a bound from above for each term in the right-hand side.

$$
\begin{aligned}
& 2 \delta t d\left(\delta_{t} u^{k+1}, u^{k+1}, \tilde{e}_{h}^{k+1}\right) \leq c \delta t\left\|\delta_{t} u^{k+1}\right\|_{0}\left\|u^{k+1}\right\|_{2}\left\|\tilde{e}_{h}^{k+1}\right\|_{1} \\
& \leq c \delta t^{2}\left\|u_{t}\right\|_{L^{\infty}\left(L^{2}\right)}\|u\|_{L^{\infty}\left(H^{2}\right)}\left\|\tilde{e}_{h}^{k+1}\right\|_{1} \\
& \leq c_{\gamma} \delta t^{3}+\gamma \delta t\left\|\tilde{e}_{h}^{k+1}\right\|_{1}^{2} . \\
& 2 \delta t d\left(u^{k}-w_{h}^{k}, u^{k+1}, \tilde{e}_{h}^{k+1}\right) \\
& \leq c \delta t\left\|u^{k}-w_{h}^{k}\right\|_{0}\left\|u^{k+1}\right\|_{2}\left\|\tilde{e}_{h}^{k+1}\right\|_{1} \\
& \leq c \delta t h^{l+1}\left(\|u\|_{L^{\infty}\left(H^{l+1}\right)}+\|p\|_{L^{\infty}\left(H^{l}\right)}\right)\left\|\tilde{e}_{h}^{k+1}\right\|_{1} \\
& \leq c_{\gamma} \delta t h^{2(l+1)}+\gamma \delta t\left\|\tilde{e}_{h}^{k+1}\right\|_{1}^{2} . \\
& 2 \delta t d\left(w_{h}^{k}, u^{k+1}-w_{h}^{k+1}, \tilde{e}_{h}^{k+1}\right) \\
& \leq c \delta t\left(\left\|w_{h}^{k}\right\|_{1,3}+\left\|w_{h}^{k}\right\|_{0, \infty}\right)\left\|u^{k+1}-w_{h}^{k+1}\right\|_{0,2}\left\|\tilde{e}_{h}^{k+1}\right\|_{1,2} \\
& \leq c \delta t h^{l+1}\left(\|u\|_{L^{\infty}\left(H^{l+1}\right)}+\|p\|_{L^{\infty}\left(H^{l}\right)}\right)\left\|\tilde{e}_{h}^{k+1}\right\|_{1,2} \\
& \leq c_{\gamma} \delta t h^{2(l+1)}+\gamma \delta t\left\|\tilde{e}_{h}^{k+1}\right\|_{1}^{2} .
\end{aligned}
$$

Using the inequality $\left\|\tilde{e}_{h}^{k+1}\right\|_{0} \leq\left\|\tilde{e}_{h}^{k+1}-i_{h}^{\mathrm{T}} e_{h}^{k}\right\|_{0}+\left\|e_{h}^{k}\right\|_{0}$, we deduce

$$
\begin{aligned}
2 \delta t d\left(\tilde{e}_{h}^{k}, w_{h}^{k+1}, \tilde{e}_{h}^{k+1}\right) & \leq c \delta t\left\|\tilde{e}_{h}^{k}\right\|_{1,2}\left(\left\|w_{h}^{k}\right\|_{1,3}+\left\|w_{h}^{k}\right\|_{0, \infty}\right)\left\|\tilde{e}_{h}^{k+1}\right\|_{0,2} \\
& \leq c \delta t\left(\|u\|_{L^{\infty}\left(H^{2}\right)}+\|p\|_{L^{\infty}\left(H^{1}\right)}\right)\left\|\tilde{e}_{h}^{k}\right\|_{1}\left\|\tilde{e}_{h}^{k+1}\right\|_{0} \\
& \leq c_{\gamma} \delta t\left\|\tilde{e}_{h}^{k+1}\right\|_{0}^{2}+\gamma \delta t\left\|\tilde{e}_{h}^{k}\right\|_{1}^{2} \cdot \\
& \leq c_{\gamma} \delta t\left\|e_{h}^{k}\right\|_{0}^{2}+c_{\gamma} \delta t\left\|\tilde{e}_{h}^{k+1}-i_{h}^{\mathrm{T}} e_{h}^{k}\right\|_{0}^{2}+\gamma \delta t\left\|\tilde{e}_{h}^{k}\right\|_{1}^{2} .
\end{aligned}
$$

Numerische Mathematik Electronic Edition page 229 of Numer. Math. (1998) 80: 207-238 
Given the skew-symmetry of $d$, the last term $d\left(\tilde{u}_{h}^{k}, \tilde{e}_{h}^{k+1}, \tilde{e}_{h}^{k+1}\right)$ is zero.

After collecting all the bounds concerning the residuals, we obtain:

$$
\begin{aligned}
2 \delta t\left\langle R^{k+1}+\right. & \left.R_{\mathrm{n} .1 .}^{k+1}, \tilde{e}_{h}^{k+1}\right\rangle \\
\leq & c_{\gamma} \delta t\left\|e_{h}^{k}\right\|_{0}^{2}+c_{\gamma} \delta t\left\|\tilde{e}_{h}^{k+1}-i_{h}^{\mathrm{T}} e_{h}^{k}\right\|_{0}^{2} \\
& +\gamma \delta t\left\|\tilde{e}_{h}^{k}\right\|_{1}^{2}+4 \gamma \delta t\left\|\tilde{e}_{h}^{k+1}\right\|_{1}^{2}+c_{\gamma}^{\prime} \delta t\left(\delta t+h^{l+1}\right)^{2} .
\end{aligned}
$$

By combining the bounds obtained above, we infer

$$
\begin{aligned}
& \left\|\tilde{e}_{h}^{k+1}\right\|_{0}^{2}+\left(1-c_{\gamma} \delta t\right)\left\|\tilde{e}_{h}^{k+1}-i_{h}^{\mathrm{T}} e_{h}^{k}\right\|_{0}^{2} \\
& +(2 \alpha-4 \gamma) \delta t\left\|\tilde{e}_{h}^{k+1}\right\|_{1}^{2}+2 \delta t\left(\tilde{e}_{h}^{k+1}, B_{h}^{\mathrm{T}} \psi_{h}^{k}\right) \\
& \quad \leq\left\|e_{h}^{k}\right\|_{0}^{2}+c_{\gamma} \delta t\left\|e_{h}^{k}\right\|_{0}^{2}+\gamma \delta t\left\|\tilde{e}_{h}^{k}\right\|_{1}^{2}+c_{\gamma}^{\prime} \delta t\left(\delta t+h^{l+1}\right)^{2} .
\end{aligned}
$$

Now we choose $\gamma=\alpha / 5$ and $\delta t \leq 1 /\left(2 c_{\gamma}\right)$; the inequality above becomes

$$
\left\|\tilde{e}_{h}^{k+1}\right\|_{0}^{2}+\frac{1}{2}\left\|\tilde{e}_{h}^{k+1}-i_{h}^{\mathrm{T}} e_{h}^{k}\right\|_{0}^{2}+\frac{6}{5} \alpha \delta t\left\|\tilde{e}_{h}^{k+1}\right\|_{1}^{2}+2 \delta t\left(\tilde{e}_{h}^{k+1}, B_{h}^{\mathrm{T}} \psi_{h}^{k}\right)
$$

(c) To have some control on $2 \delta t\left(\tilde{e}_{h}^{k+1}, B_{h}^{\mathrm{T}} \epsilon_{h}^{k+1}\right)$, we take the inner product of the first equation of (5.13) by $2 \delta t^{2} C_{h}^{\mathrm{T}} \psi_{h}^{k}$ and we obtain,

$$
\begin{aligned}
-2 \delta t\left(\tilde{e}_{h}^{k+1}, B_{h}^{\mathrm{T}} \psi_{h}^{k}\right) & +\delta t^{2}\left\|C_{h}^{\mathrm{T}} \epsilon_{h}^{k+1}\right\|_{0}^{2}-\left\|e_{h}^{k+1}-i_{h} \tilde{e}_{h}^{k+1}\right\|_{0}^{2} \\
& =\delta t^{2}\left\|C_{h}^{\mathrm{T}} \psi_{h}^{k}\right\|_{0}^{2} \\
& =\delta t^{2}\left\|C_{h}^{\mathrm{T}}\left(\delta_{t} q_{h}^{k+1}+\epsilon_{h}^{k}\right)\right\|_{0}^{2},
\end{aligned}
$$

that is to say, given the stability result of Lemma 5.3, we have

$$
\begin{aligned}
-2 \delta t\left(\tilde{e}_{h}^{k+1}, B_{h}^{\mathrm{T}} \psi_{h}^{k}\right)+ & \delta t^{2}\left\|C_{h}^{\mathrm{T}} \epsilon_{h}^{k+1}\right\|_{0}^{2}-\left\|e_{h}^{k+1}-i_{h} \tilde{e}_{h}^{k+1}\right\|_{0}^{2} \\
\leq & \delta t^{2}(1+\delta t)\left\|C_{h}^{\mathrm{T}} \epsilon_{h}^{k}\right\|_{0}^{2} \\
& +c \delta t^{3}\left(\frac{1}{4}+\delta t\right)\left(\left\|u_{t}\right\|_{L^{\infty}\left(H^{2}\right)}+\left\|p_{t}\right\|_{L^{\infty}\left(H^{1}\right)}\right) \\
\leq & \delta t^{2}(1+\delta t)\left\|C_{h}^{\mathrm{T}} \epsilon_{h}^{k}\right\|_{0}^{2}+c \delta t^{3} .
\end{aligned}
$$

(d) We obtain some control on $e_{h}^{k+1}$ by taking the inner product of (5.13) by $2 \delta t e_{h}^{k+1}$

$$
\left\|e_{h}^{k+1}\right\|_{0}^{2}+\left\|e_{h}^{k+1}-i_{h} \tilde{e}_{h}^{k+1}\right\|_{0}^{2}-\left\|\tilde{e}_{h}^{k+1}\right\|_{0}^{2}=0 .
$$

(e) After summing up (5.14) + (5.15) + (5.16) we obtain

$$
\begin{gathered}
\left\|e_{h}^{k+1}\right\|_{0}^{2}+\delta t^{2}\left\|C_{h}^{\mathrm{T}} \epsilon_{h}^{k+1}\right\|_{0}^{2}+\frac{6 \alpha}{5} \delta t\left\|\tilde{e}_{h}^{k+1}\right\|_{1}^{2}+\frac{1}{2}\left\|\tilde{e}_{h}^{k+1}-i_{h}^{\mathrm{T}} e_{h}^{k}\right\|_{0}^{2} \\
\leq\left(1+c_{1} \delta t\right)\left(\left\|e_{h}^{k}\right\|_{0}^{2}+\delta t^{2}\left\|C_{h}^{\mathrm{T}} \epsilon_{h}^{k}\right\|_{0}^{2}\right)+\frac{\alpha}{5} \delta t\left\|\tilde{e}_{h}^{k}\right\|_{1}^{2}+c_{2} \delta t\left(\delta t+h^{l+1}\right)^{2},
\end{gathered}
$$

Numerische Mathematik Electronic Edition page 230 of Numer. Math. (1998) 80: 207-238 
where constants $c_{1}$ and $c_{2}$ depend on the data of the problem. By taking the sum from $k=k_{0}$ to some integer $n \leq K$ we obtain

$$
\begin{gathered}
\left\|e_{h}^{n+1}\right\|_{0}^{2}+\delta t^{2}\left\|C_{h}^{\mathrm{T}} \epsilon_{h}^{n+1}\right\|_{0}^{2}+\alpha \delta t \sum_{k=k_{0}}^{n}\left\|\tilde{e}_{h}^{k+1}\right\|_{1}^{2} \\
+\frac{\alpha}{5} \delta t\left\|\tilde{e}_{h}^{n+1}\right\|_{1}^{2}+\frac{1}{2} \sum_{k=k_{0}}^{n}\left\|\tilde{e}_{h}^{k+1}-i_{h}^{\mathrm{T}} e_{h}^{k}\right\|_{0}^{2} \\
\leq\left\|e_{h}^{k_{0}}\right\|_{0}^{2}+\delta t^{2}\left\|C_{h}^{\mathrm{T}} \epsilon_{h}^{k_{0}}\right\|_{0}^{2}+\frac{\alpha}{5} \delta t\left\|\tilde{e}_{h}^{k_{0}}\right\|_{1}^{2}+c_{2}\left(\delta t+h^{l+1}\right)^{2} \\
+c_{1} \delta t \sum_{k=k_{0}}^{n}\left(\left\|e_{h}^{k}\right\|_{0}^{2}+\delta t^{2}\left\|C_{h}^{\mathrm{T}} \epsilon_{h}^{k}\right\|_{0}^{2}\right) .
\end{gathered}
$$

By our particular choice of the initial conditions and by using the discrete Gronwall Lemma 5.4, the final result is

(5.17) $\left\|e_{h}^{n+1}\right\|_{0}^{2}+\alpha \delta t \sum_{k=k_{0}}^{n+1}\left\|\tilde{e}_{h}^{k}\right\|_{1}^{2}+\sum_{k=k_{0}}^{n}\left\|\tilde{e}_{h}^{k+1}-i_{h}^{\mathrm{T}} e_{h}^{k}\right\|_{0}^{2} \leq c\left(\delta t+h^{l+1}\right)^{2}$.

Furthermore, since we have

$u\left(t^{k}\right)-u_{h}^{k}=e_{h}^{k}+u\left(t^{k}\right)-w_{h}\left(t^{k}\right), \quad u\left(t^{k}\right)-\tilde{u}_{h}^{k}=\tilde{e}_{h}^{k}+u\left(t^{k}\right)-w_{h}\left(t^{k}\right)$, we obtain the desired result by using the interpolation properties of $w_{h}$. Note that the convergence result on $\tilde{u}_{h}$ in the $l^{\infty}\left(L^{2}(\Omega)^{d}\right)$ norm is a consequence of

$$
\begin{aligned}
\left\|\tilde{e}_{h}^{k+1}\right\|_{0} & \leq\left\|\tilde{e}_{h}^{k+1}-i_{h}^{\mathrm{T}} e_{h}^{k}\right\|_{0}+\left\|e_{h}^{k}\right\|_{0} \\
& \leq c\left(\delta t+h^{l+1}\right) .
\end{aligned}
$$

Remark 5.1. Note that although the approximation error $\left\|\tilde{e}_{h}^{m+1}\right\|_{l^{2}\left(H^{1}(\Omega)^{d}\right)}$ is of order $\mathcal{O}\left(t+h^{l+1}\right)$, the global error is spoiled by the interpolation error in space which is of order $\mathcal{O}\left(h^{l}\right)$.

In addition to the hypotheses of Theorem 5.5, we assume also that $u_{t t} \in L^{\infty}\left(H^{l+1}(\Omega)^{d}\right), u_{t t t} \in L^{\infty}\left(H^{1}(\Omega)^{d}\right)$, and $p_{t t} \in L^{\infty}\left(H^{l}(\Omega)^{d}\right)$, $p_{t t t} \in L^{\infty}\left(L^{2}(\Omega)\right)$. Furthermore we assume that the projection algorithm is initialized so that

$$
\begin{gathered}
\left\|e_{h}^{k_{0}}\right\|_{0} \leq c \delta t\left(\delta t+h^{l}\right), \\
\left\|\tilde{e}_{h}^{k_{0}}\right\|_{1} \leq c \delta t^{1 / 2}\left(\delta t+h^{l}\right), \\
\left\|C_{h}^{\mathrm{T}} \epsilon_{h}^{k_{0}}\right\|_{0} \leq c\left(\delta t+h^{l}\right) .
\end{gathered}
$$

Then, the ability of $\delta_{t} u_{h}^{k+1} / \delta t$ to approximate $d w_{h}\left(t^{k+1}\right) / d t$ is made explicit by

Numerische Mathematik Electronic Edition page 231 of Numer. Math. (1998) 80: 207-238 
Lemma 5.6. Assume $\delta t \leq c_{\mathrm{S}} /\left(1+\left|\log \left(h^{-1}\right)\right|\right)^{1 / 2}$ in $2 D$ or $\delta t \leq c_{\mathrm{S}} h^{\frac{1}{2}}$ in $3 D$; under the hypotheses above and if $c_{\mathrm{S}}$ is small enough, the solution to the projection scheme (3.3)-(3.4) satisfies:

$$
\left\|\delta_{t} e_{h}^{k}\right\|_{l^{\infty}\left(L^{2}(\Omega)^{d}\right)}+\left\|\delta_{t} \tilde{e}_{h}^{k}\right\|_{l^{2}\left(H^{1}(\Omega)^{d}\right)} \leq c \delta t\left(\delta t+h^{l}\right) .
$$

Proof. (a) First we control the first time step: $\delta_{t} \tilde{e}_{h}^{k_{0}+1}, \delta_{t} e_{h}^{k_{0}+1}$, and $\delta_{t} \epsilon_{h}^{k_{0}+1}$. We note that

$$
\begin{aligned}
\left\|\tilde{e}_{h}^{k_{0}+1}\right\|_{0}^{2} & +2 \alpha \delta t\left\|\tilde{e}_{h}^{k_{0}+1}\right\|_{1}^{2} \\
& \leq\left\|e_{h}^{k_{0}}\right\|_{0}^{2}-2 \delta t\left(\tilde{e}_{h}^{k_{0}+1}, B_{h}^{\mathrm{T}} \psi_{h}^{k_{0}}\right)+2 \delta t\left\langle R^{k_{0}+1}+R_{\text {n. l. }}^{k_{0}+1}, \tilde{e}_{h}^{k_{0}+1}\right\rangle .
\end{aligned}
$$

Using the assumed regularity of $u$ and $p$ for $t \geq t_{0}$, we have

$$
\begin{aligned}
2 \delta t\left(R^{k_{0}+1}, \tilde{e}_{h}^{k_{0}+1}\right) & \leq \gamma\left\|\tilde{e}_{h}^{k_{0}+1}\right\|_{0}^{2}+c_{\gamma} \delta t^{2}\left\|R^{k_{0}+1}\right\|_{0}^{2} \\
& \leq \gamma\left\|\tilde{e}_{h}^{k_{0}+1}\right\|_{0}^{2}+c_{\gamma} \delta t^{2}\left(\delta t+h^{l+1}\right)^{2} .
\end{aligned}
$$

We now give bounds from above for the nonlinear terms.

$$
\begin{gathered}
2 \delta t d\left(\delta_{t} u^{k_{0}+1}, u^{k_{0}+1}, \tilde{e}_{h}^{k_{0}+1}\right) \leq c \delta t\left\|\delta_{t} u^{k_{0}+1}\right\|_{1}\left\|u^{k_{0}+1}\right\|_{2}\left\|\tilde{e}_{h}^{k_{0}+1}\right\|_{0} \\
\leq c_{\gamma} \delta t^{4}+\gamma\left\|\tilde{e}_{h}^{k_{0}+1}\right\|_{0}^{2} . \\
2 \delta t d\left(u^{k_{0}}-w_{h}^{k_{0}}, u^{k_{0}+1}, \tilde{e}_{h}^{k_{0}+1}\right) \leq c \delta t\left\|u^{k_{0}}-w_{h}^{k_{0}}\right\|_{1}\left\|u^{k_{0}+1}\right\|_{2}\left\|\tilde{e}_{h}^{k_{0}+1}\right\|_{0} \\
\leq c_{\gamma} \delta t^{2} h^{2 l}+\gamma\left\|\tilde{e}_{h}^{k_{0}+1}\right\|_{0}^{2} . \\
2 \delta t d\left(w_{h}^{k_{0}}, u^{k_{0}+1}-w_{h}^{k_{0}+1}, \tilde{e}_{h}^{k_{0}+1}\right) \\
\leq c \delta t\left(\left\|w_{h}^{k_{0}}\right\|_{0, \infty}+\left\|w_{h}^{k_{0}}\right\|_{1,3}\right)\left\|u^{k_{0}+1}-w_{h}^{k_{0}+1}\right\|_{1,2}\left\|\tilde{e}_{h}^{k_{0}+1}\right\|_{0,2} \\
\leq c_{\gamma} \delta t^{2} h^{2 l}+\gamma\left\|\tilde{e}_{h}^{k_{0}+1}\right\|_{0}^{2} \cdot \\
2 \delta t d\left(\tilde{e}_{h}^{k_{0}}, w_{h}^{k_{0}+1}, \tilde{e}_{h}^{k_{0}+1}\right) \\
\leq c \delta t\left\|\tilde{e}_{h}^{k_{0}}\right\|_{1,2}\left(\left\|w_{h}^{k_{0}+1}\right\|_{0, \infty}+\left\|w_{h}^{k_{0}+1}\right\|_{1,3}\right)\left\|\tilde{e}_{h}^{k_{0}+1}\right\|_{0} \\
\leq \gamma\left\|\tilde{e}_{h}^{k_{0}+1}\right\|_{0}^{2}+c_{\gamma} \delta t^{2}\left\|\tilde{e}_{h}^{k_{0}}\right\|_{1}^{2} \\
\leq c_{\gamma} \delta t^{2}\left(\delta t+h^{l}\right)^{2}+\gamma\left\|\tilde{e}_{h}^{k_{0}+1}\right\|_{0}^{2} .
\end{gathered}
$$

After collecting all the bounds involving the residuals, we obtain

$$
2 \delta t\left\langle R^{k_{0}+1}+R_{\text {n. l. }}^{k_{0}+1}, \tilde{e}_{h}^{k_{0}+1}\right\rangle \leq 5 \gamma\left\|\tilde{e}_{h}^{k_{0}+1}\right\|_{0}^{2}+c_{\gamma} \delta t^{2}\left(\delta t+h^{l}\right)^{2} .
$$


As a result, we have

$$
\begin{aligned}
\left\|\tilde{e}_{h}^{k_{0}+1}\right\|_{0}^{2}+ & 2 \alpha \delta t\left\|\tilde{e}_{h}^{k_{0}+1}\right\|_{1}^{2} \\
\leq & \left\|e_{h}^{k_{0}}\right\|_{0}^{2}-2 \delta t\left(\tilde{e}_{h}^{k_{0}+1}, B_{h}^{\mathrm{T}} \psi_{h}^{k_{0}}\right) \\
& \quad+5 \gamma\left\|\tilde{e}_{h}^{k_{0}+1}\right\|_{0}^{2}+c_{\gamma} \delta t^{2}\left(\delta t+h^{l}\right)^{2} \\
\leq & -2 \delta t\left(\tilde{e}_{h}^{k_{0}+1}, B_{h}^{\mathrm{T}}\left[\delta_{t} q_{h}^{k_{0}+1}+\epsilon_{h}^{k_{0}}\right]\right) \\
& \quad+5 \gamma\left\|\tilde{e}_{h}^{k_{0}+1}\right\|_{0}^{2}+c_{\gamma} \delta t^{2}\left(\delta t+h^{l}\right)^{2} \\
\leq & 6 \gamma\left\|\tilde{e}_{h}^{k_{0}+1}\right\|_{0}^{2}+c_{\gamma}^{\prime} \delta t^{2}\left\|C_{h}^{\mathrm{T}} \epsilon_{h}^{k_{0}}\right\|_{0}^{2}+c_{\gamma} \delta t^{2}\left(\delta t+h^{l}\right)^{2} \\
\leq & 6 \gamma\left\|\tilde{e}_{h}^{k_{0}+1}\right\|_{0}^{2}+c_{\gamma} \delta t^{2}\left(\delta t+h^{l}\right)^{2} .
\end{aligned}
$$

We choose $\gamma=1 / 12$; as a result, we obtain the bound

$$
\left\|\tilde{e}_{h}^{k_{0}+1}\right\|_{0}^{2}+2 \alpha \delta t\left\|\tilde{e}_{h}^{k_{0}+1}\right\|_{1}^{2} \leq c \delta t^{2}\left(\delta t+h^{l}\right)^{2} .
$$

That is to say

$$
\left\|\delta_{t} \tilde{e}_{h}^{k_{0}+1}\right\|_{0} \leq c \delta t\left(\delta t+h^{l}\right), \quad\left\|\delta_{t} \tilde{e}_{h}^{k_{0}+1}\right\|_{1} \leq c \delta t^{1 / 2}\left(\delta t+h^{l}\right) .
$$

Furthermore, from the projection step (5.13) we obtain

$$
\left\{\begin{aligned}
\left\|e_{h}^{k_{0}+1}\right\|_{0} & \leq\left\|\tilde{e}_{h}^{k_{0}+1}\right\|_{0}, \\
\left\|C_{h}^{\mathrm{T}}\left(\epsilon_{h}^{k_{0}+1}-\psi_{h}^{k_{0}}\right)\right\|_{0} & \leq\left\|\tilde{e}_{h}^{k_{0}+1}\right\|_{0} / \delta t .
\end{aligned}\right.
$$

The first bound yields easily

$$
\left\|\delta_{t} e_{h}^{k_{0}+1}\right\|_{0} \leq c \delta t\left(\delta t+h^{l}\right) .
$$

The other bound yields

$$
\begin{aligned}
\left\|C_{h}^{\mathrm{T}}\left(\epsilon_{h}^{k_{0}+1}-\epsilon_{h}^{k_{0}}\right)\right\|_{0} & \leq\left\|\tilde{e}_{h}^{k_{0}+1}\right\|_{0} / \delta t+\left\|C_{h}^{\mathrm{T}} \delta_{t} q_{h}^{k_{0}+1}\right\|_{0} \\
& \leq c\left(\delta t+h^{l}\right) .
\end{aligned}
$$

In other words, we have

$$
\left\|C_{h}^{\mathrm{T}} \delta_{t} \epsilon_{h}^{k_{0}+1}\right\|_{0} \leq c\left(\delta t+h^{l}\right) .
$$

(b) Now we proceed as in the proof of Theorem 5.5. For $k \geq k_{0}+1$, the equation that controls the error $\delta_{t} \tilde{e}_{h}^{k+1}$ is

$$
\frac{\delta_{t} \tilde{e}_{h}^{k+1}-i_{h}^{\mathrm{T}} \delta_{t} e_{h}^{k}}{\delta t}+A_{h} \delta_{t} \tilde{e}_{h}^{k+1}+B_{h}^{\mathrm{T}} \delta_{t} \psi_{h}^{k}=i_{X_{h}}^{\mathrm{T}}\left[\delta_{t} R^{k+1}+\delta_{t} R_{\text {n. } 1 .}^{k+1}\right] \text {, }
$$

and the system of equations that controls $\delta_{t} e_{h}^{k}$ and $\delta_{t} \epsilon_{h}^{k}$ is found to be

$$
\left\{\begin{array}{l}
\frac{\delta_{t} e_{h}^{k+1}-i_{h} \delta_{t} \tilde{e}_{h}^{k+1}}{\delta t}+C_{h}^{\mathrm{T}}\left[\delta_{t} \epsilon_{h}^{k+1}-\delta_{t} \psi_{h}^{k}\right]=0 \\
C_{h} \delta_{t} e_{h}^{k+1}=0
\end{array}\right.
$$

Numerische Mathematik Electronic Edition page 233 of Numer. Math. (1998) 80: 207-238 
Given the assumed regularity of $u$ and $p$, we have

$$
\begin{aligned}
& \left\langle\delta_{t} R^{k+1}, \delta_{t} \tilde{e}_{h}^{k+1}\right\rangle \\
& \leq \gamma\left\|\delta_{t} \tilde{e}_{h}^{k+1}\right\|_{1}^{2}+c \delta t^{2}\left(\delta t\left\|u_{t t t}\right\|_{L^{\infty}\left(H^{1}\right)}+h^{l+1}\left\|u_{t t}\right\|_{L^{\infty}\left(H^{l+1}\right)}\right)^{2} \\
& \leq \gamma\left\|\delta_{t} \tilde{e}_{h}^{k+1}\right\|_{1}^{2}+c \delta t^{2}\left(\delta t+h^{l+1}\right)^{2} .
\end{aligned}
$$

Furthermore, the nonlinear term is decomposed as follows

$$
\begin{aligned}
-\left\langle\delta_{t}\right. & \left.R_{\mathrm{n} .1 .}^{k+1}, \delta_{t} \tilde{e}_{h}^{k+1}\right\rangle \\
= & -\left\langle D_{h}\left(\tilde{u}_{h}^{k}, \tilde{u}_{h}^{k+1}\right)-D_{h}\left(\tilde{u}_{h}^{k-1}, \tilde{u}_{h}^{k}\right)\right. \\
& \left.\quad-D\left(u_{h}^{k+1}, u_{h}^{k+1}\right)+D\left(u_{h}^{k}, u_{h}^{k}\right), \delta_{t} \tilde{e}_{h}^{k+1}\right\rangle \\
= & d\left(\tilde{u}_{h}^{k}, \tilde{e}_{h}^{k+1}, \delta_{t} \tilde{e}_{h}^{k+1}\right)+d\left(\tilde{e}_{h}^{k}, \delta_{t} w_{h}^{k+1}, \delta_{t} \tilde{e}_{h}^{k+1}\right) \\
& +d\left(\delta_{t} w_{h}^{k+1}, \delta_{t} w_{h}^{k+1}, \delta_{t} \tilde{e}_{h}^{k+1}\right)+d\left(w_{h}^{k+1}, \delta_{t}\left(u^{k+1}-w_{h}^{k+1}\right), \delta_{t} \tilde{e}_{h}^{k+1}\right) \\
& +d\left(u^{k+1}-w_{h}^{k+1}, \delta_{t} u^{k+1}, \delta_{t} \tilde{e}_{h}^{k+1}\right)+d\left(\delta_{t} \tilde{e}_{h}^{k}, \tilde{u}_{h}^{k}, \delta_{t} \tilde{e}_{h}^{k+1}\right) \\
& +d\left(\delta_{t} w_{h}^{k}, \tilde{e}_{h}^{k}, \delta_{t} \tilde{e}_{h}^{k+1}\right)+d\left(\delta_{t t} w_{h}^{k+1}, w_{h}^{k}, \delta_{t} \tilde{e}_{h}^{k+1}\right) \\
& +d\left(\delta_{t}\left(u^{k+1}-w_{h}^{k+1}\right), w_{h}^{k}, \delta_{t} \tilde{e}_{h}^{k+1}\right)+d\left(\delta_{t} u^{k+1}, u^{k}-w_{h}^{k}, \delta_{t} \tilde{e}_{h}^{k+1}\right) .
\end{aligned}
$$

Every term is easy to bound from above except the first one and the sixth one. For the sixth term we have

$$
\begin{aligned}
& d\left(\delta_{t} \tilde{e}_{h}^{k}, \tilde{u}_{h}^{k}, \delta_{t} \tilde{e}_{h}^{k+1}\right) \\
& =d\left(\delta_{t} \tilde{e}_{h}^{k}, \tilde{u}_{h}^{k}-w_{h}^{k}, \delta_{t} \tilde{e}_{h}^{k+1}\right)+d\left(\delta_{t} \tilde{e}_{h}^{k}, w_{h}^{k}, \delta_{t} \tilde{e}_{h}^{k+1}\right) \\
& \leq c_{1}\left\|\tilde{e}_{h}^{k}\right\|_{0,2}\left(\left\|\delta_{t} \tilde{e}_{h}^{k}\right\|_{0, \infty}\left\|\delta_{t} \tilde{e}_{h}^{k+1}\right\|_{1,2}+\left\|\delta_{t} \tilde{e}_{h}^{k}\right\|_{1,2}\left\|\delta_{t} \tilde{e}_{h}^{k+1}\right\|_{0, \infty}\right) \\
& \quad \quad+c_{2}\left(\left\|w_{h}^{k}\right\|_{0, \infty}+\left\|w_{h}^{k}\right\|_{1,3}\right)\left\|\delta_{t} \tilde{e}_{h}^{k}\right\|_{0,2}\left\|\delta_{t} \tilde{e}_{h}^{k+1}\right\|_{1,2} \\
& \quad \leq c_{1} c(h)\left\|\tilde{e}_{h}^{k}\right\|_{0}\left\|\delta_{t} \tilde{e}_{h}^{k}\right\|_{1}\left\|\delta_{t} \tilde{e}_{h}^{k+1}\right\|_{1}+c_{2}\left\|\delta_{t} \tilde{e}_{h}^{k}\right\|_{0}\left\|\delta_{t} \tilde{e}_{h}^{k+1}\right\|_{1} \\
& \leq c_{1} c(h)\left(\delta t+h^{l+1}\right)\left\|\delta_{t} \tilde{e}_{h}^{k}\right\|_{1}\left\|\delta_{t} \tilde{e}_{h}^{k+1}\right\|_{1}+c_{2}\left\|\delta_{t} \tilde{e}_{h}^{k}\right\|_{0}\left\|\delta_{t} \tilde{e}_{h}^{k+1}\right\|_{1},
\end{aligned}
$$

where $c(h)$ denotes the constant appearing in the following classical inverse inequality

$$
\left\|\phi_{h}\right\|_{0, \infty} \leq\left\{\begin{array}{l}
c\left(1+\left|\log \left(h^{-1}\right)\right|\right)^{1 / 2}\left\|\phi_{h}\right\|_{1,2}, \quad \text { in 2D } \\
c h^{-1 / 2}\left\|\phi_{h}\right\|_{1,2}, \quad \text { in 3D }
\end{array}\right.
$$

Assuming $\delta t \leq c_{\mathrm{S}} /\left(1+\left|\log \left(h^{-1}\right)\right|\right)^{1 / 2}$ in $2 \mathrm{D}$ or $\delta t \leq c_{\mathrm{S}} h^{1 / 2}$ in $3 \mathrm{D}$ and choosing $c_{\mathrm{S}}$ and $h$ small enough we obtain

$$
d\left(\delta_{t} \tilde{e}_{h}^{k}, \tilde{u}_{h}^{k}, \delta_{t} \tilde{e}_{h}^{k+1}\right) \leq \frac{\gamma}{10}\left\|\delta_{t} \tilde{e}_{h}^{k}\right\|_{1}^{2}+\frac{\gamma}{10}\left\|\delta_{t} \tilde{e}_{h}^{k+1}\right\|_{1}^{2}+c_{\gamma}\left\|\delta_{t} \tilde{e}_{h}^{k}\right\|_{0}^{2}
$$

Numerische Mathematik Electronic Edition page 234 of Numer. Math. (1998) 80: 207-238 
The first term of the nonlinear residual is bounded similarly. As a result, the final bound on the nonlinear residual is

$$
\begin{gathered}
2 \delta t\left\langle\delta_{t} R_{\text {n. l. }}^{k+1}, \delta_{t} \tilde{e}_{h}^{k+1}\right\rangle \\
\leq \gamma \delta t\left\|\delta_{t} \tilde{e}_{h}^{k}\right\|_{1}^{2}+\gamma \delta t\left\|\delta_{t} \tilde{e}_{h}^{k+1}\right\|_{1}^{2}+c_{\gamma}\left\|\delta_{t} \tilde{e}_{h}^{k}\right\|_{0}^{2}+c_{\gamma}^{\prime} \delta t^{3}\left(\delta t+h^{l}\right)^{2} .
\end{gathered}
$$

(c) By reasoning as in the proof of Theorem 5.5, we see that the final bound is a consequence of (a) and (b).

Theorem 5.7. Under the hypotheses of Theorem 5.5 and Lemma 5.6, the approximate pressure given by the projection scheme (3.3)-(3.4) satisfies:

$$
\left\|p\left(t^{k}\right)-p_{h}^{k}\right\|_{l^{2}\left(L^{2}(\Omega)\right)} \leq c\left(\delta t+h^{l}\right) .
$$

Proof. By summing (5.12) and $i_{h}^{\mathrm{T}}(5.13)$ we obtain

$$
B_{h}^{\mathrm{T}} \epsilon_{h}^{k+1}=-\frac{i_{h}^{\mathrm{T}} \delta_{t} e_{h}^{k+1}}{\delta t}-A_{h} \tilde{e}_{h}^{k+1}+i_{X_{h}}^{\mathrm{T}}\left[R^{k+1}+R_{\mathrm{n} .1 .}^{k+1}\right] .
$$

The inf-sup condition yields

$c_{1}\left\|\epsilon_{h}^{k+1}\right\|_{0} \leq \frac{\left\|\delta_{t} e_{h}^{k+1}\right\|_{0}}{\delta t}+c_{2}\left\|\tilde{e}_{h}^{k+1}\right\|_{1}+\sup _{v_{h} \in X,\left\|v_{h}\right\|_{1}=1}\left\langle R^{k+1}+R_{\text {n.l. }}^{k+1}, v_{h}\right\rangle$.

That is to say

$$
c_{1}\left\|\epsilon_{h}^{k+1}\right\|_{0} \leq \frac{\left\|\delta_{t} e_{h}^{k+1}\right\|_{0}}{\delta t}+c_{2}\left\|\tilde{e}_{h}^{k+1}\right\|_{1}+c_{3}\left\|\tilde{e}_{h}^{k}\right\|_{1}+c_{4}\left(\delta t+h^{l}\right) .
$$

The final bound is a consequence of this inequality and (5.17), (5.19) together with the identity

$$
p\left(t^{k}\right)-p_{h}^{k}=\epsilon_{h}^{k}+p\left(t^{k}\right)-q_{h}\left(t^{k}\right) . \square
$$

As a direct consequence of this result we deduce

Theorem 5.8. Under the hypotheses of Theorem 5.5 and Lemma 5.6, the approximate velocity and pressure given by the projection scheme (3.3)(3.4) satisfy:

$$
\left\|u\left(t^{k}\right)-\tilde{u}_{h}^{k}\right\|_{l^{\infty}\left(H^{1}(\Omega)^{d}\right)}+\left\|p\left(t^{k}\right)-p_{h}^{k}\right\|_{l^{\infty}\left(L^{2}(\Omega)\right)} \leq c\left(\delta t+h^{l}\right) .
$$

Proof. We give a sketch of the proof. Taking the inner product of (5.26) by $2 \delta_{t} \tilde{e}_{h}^{k+1}$ we obtain

$$
\begin{aligned}
\left\|\nabla \tilde{e}_{h}^{k+1}\right\|_{0}^{2}+\left\|\nabla \delta_{t} \tilde{e}_{h}^{k+1}\right\|_{0}^{2} & \leq\left\|\nabla \tilde{e}_{h}^{k}\right\|_{0}^{2}+\delta t\left\|\epsilon_{h}^{k+1}\right\|_{0}^{2}+\frac{1}{\delta t}\left\|\nabla \delta_{t} \tilde{e}_{h}^{k+1}\right\|_{0}^{2} \\
& +\frac{2}{\delta t}\left\|\delta_{t} e_{h}^{k+1}\right\|_{0}^{2}+\left\langle R^{k+1}+R_{\text {n. l. }}^{k+1}, 2 \delta_{t} \tilde{e}_{h}^{k+1}\right\rangle .
\end{aligned}
$$

The residual terms are bounded from above as in the proof of Theorem 5.5. The convergence result on the velocity is a consequence of the discrete Gronwall Lemma together with the results of Lemma 5.6 and Theorem 5.5. The convergence result on the pressure follows easily from (5.26).

Numerische Mathematik Electronic Edition page 235 of Numer. Math. (1998) 80: 207-238 


\section{Conclusions}

Fractional-step projection techniques are simple to implement: in practice they amount to solving at each time step a convection-diffusion problem and either a Darcy or a Poisson problem. Appropriate functional settings must accommodate two different spaces for representing the two velocity fields calculated in the viscous and the incompressible half steps. In practice, the projected velocity can be completely eliminated from the algorithm and the intermediate velocity is the only one of interest.

These techniques are fast: the amount of computation is much lower than that required by coupled techniques such as those that are based on the Uzawa operator. If implemented correctly, the projection techniques are very robust - a very desirable feature for industrial applications (see Guermond and Quartapelle [19]). They yield first order accuracy in time in the natural norms. The possibility of second-order accurate projection schemes in the presence of solid no-slip boundaries has not been considered in the present work but second order accuracy in time is possible and proof of convergence are reported in Guermond [18].

One argument often raised against fractional-step projection techniques is that the homogeneous Neumann boundary condition imposed at the projection step is not "physical" and generally not satisfied by the "exact" solution. On the one hand, this point is correct in the sense that the Neumann boundary condition is responsible for a limitation on the accuracy order in time (possibly second order on the velocity), but on the other hand it is not completely relevant, for the projection techniques give convergence on the pressure only in the $L^{2}(\Omega)$ norm, hence inaccurate Neumann boundary values for the pressure are theoretically admissible and should not be a major concern. In short, users of projection techniques should recall that these techniques are meaningful only in the variational sense (they are based on a Hilbertian projection). Any attempt to formulate numerical approximations of projection techniques within strong frameworks (i.e. in some strong sense) is very likely to fail and to compel the authors of such attempts to claim that projection techniques suffer from "unphysical" boundary conditions. Of course, if for some reason one desires to achieve at each time step convergence on the pressure in a much stronger norm than that of $L^{2}(\Omega)$, one certainly should not use projection techniques but should better try preconditioned Uzawa techniques.

Acknowledgements. The authors are grateful to Vivette Girault and Alfio Quarteroni for helpful discussions and remarks that improved the content of this paper. The present work has been partly supported by the Galileo Program; the support of this program is greatly acknowledged.

Numerische Mathematik Electronic Edition page 236 of Numer. Math. (1998) 80: 207-238 


\section{References}

1. Amrouche, C., Girault, V.: (1991) On the existence and regularity of the solution of Stokes problem in arbitrary dimension, Proc. Japan Acad. 67, Ser. A, 171-175

2. Azaiez, M., Bernardi, C., Grundmann, M.: (1993) Méthodes spectrales pour les équations du milieu poreux R 93029, Laboratoire d'Analyse Numérique, Paris VI

3. Babǔska, I.: (1973) The finite element method with Lagragian multipliers, Numer. Math. 20, 179-192

4. Bell, J.B., Colella, P., Glaz, H.M.: (1989) A second order projection method for the incompressible Navier-Stokes equations, J. Comput. Phys. 85, 257-283

5. Bernardi, C., Girault, V., Sanchez-Palencia, E., Private communications

6. Bernardi, C., Raugel, G.: (1985) A conforming finite element method for the timedependent Navier-Stokes equations, SIAM J. Numer. Anal. 22, 455-473

7. Brezis, H.: (1983) Analyse fonctionnelle, théorie et applications, Masson, Paris

8. Brezzi, F.: (1974) On the existence uniqueness and approximation of saddle-point problems arising from Lagrangian multipliers, R.A.I.R.O.R. 2, 129-151

9. Cattabriga, L.: (1961) Su un problema al contorno relativo al sistema di equazioni di Stokes, Rend. Sem. Mat. Univ. Padova, 31, 308-340

10. Chorin, A.J.: (1968) Numerical solution of the Navier-Stokes equations, Math. Comp. 22, 745-762

11. Chorin, A.J.: (1969) On the convergence of discrete approximations to the NavierStokes equations, Math. Comp. 23, 341-353

12. Dautray, R., Lions, J.-L.: (1984) Analyse mathématique et calcul numérique pour les sciences et les techniques, Masson, Paris

13. Donea, J., Giuliani, S., Laval, H., Quartapelle, L.: (1982) Finite element solution of the unsteady Navier-Stokes equations by a fractional step method, Comput. Meths. Appl. Mech. Eng. 30, 53-73

14. Girault, V., Raviart, P.-A.: (1986) Finite Element Methods for Navier-Stokes Equations, Springer Series in Computational Mathematics 5, Springer-Verlag

15. Gresho, P.M., Chan, S.T.: (1990) On the theory of semi-implicit projection methods for viscous incompressible flow and its implementation via finite element method that also introduces a nearly consistent mass matrix. Part I and Part II, Int. J. Numer. Methods Fluids 11, 587-620

16. Guermond, J.-L.: (1996) Some practical implementations of projection methods for Navier-Stokes equations, Modél. Math. Anal. Numér. (M² AN), 30, 5, 637-667

17. Guermond, J.-L.: (1994) Sur l'approximation des équations de Navier-Stokes instationnaires par une méthode de projection, C.R. Acad. Sci. Paris, Série I 319, 887-892

18. Guermond, J.-L.: (1997) Un résultat de convergence à l'ordre deux en temps pour l'approximation des équations de Navier-Stokes par une technique de projection, Modél. Math. Anal. Numér. $\left(\mathrm{M}^{2} \mathrm{AN}\right)$, in press, and C.R. Acad. Sci. Paris, Série I 325, 1329-1332

19. Guermond, J.-L., Quartapelle, L.: (1995) Calculation of incompressible viscous flows by an unconditionally stable projection FEM, J. Comput. Phys. 132, 1, 12-33, and Unconditionally stable finite-element method for the unsteady Navier-Stokes equations, 9th International Conference on Finite Elements in Fluids, Eds. Morandi Cecchi et al., Venezia, Italy, 16-20 October 1995, 367-376

20. Guermond, J.-L., Quartapelle, L.: (1997) On sensitive vector Poisson and Stokes problems, M3AS: Math. Mod. Meths. Appli. Sci. 7, 5

21. Heywood, J.G., Rannacher, R.: Finite element approximation of the nonstationary Navier-Stokes problem, I, II, III, and IV, SIAM J. Numer. Anal. 19 (1982) 275-311; 23 (1986) 750-777; 25 (1988) 489-512; 27 (1990) 353-384

Numerische Mathematik Electronic Edition page 237 of Numer. Math. (1998) 80: 207-238 
22. Ku, H.-C., Hirsh, R.S., Taylor, T.D.: (1987) A pseudospectral method for solution of the three-dimensional incompressible Navier-Stokes equations, J. Comput. Phys. 70, 439-462

23. Lions, J.-L., Magenes, E.: (1968) Problèmes aux limites non homogènes et applications, Dunod, Paris

24. Quartapelle, L.: (1993) Numerical Solution of the Incompressible Navier-Stokes Equations, ISNM 113, Birkhäuser, Basel

25. Quarteroni, A., Valli, A.: (1994) Numerical Approximation of Partial Differential Equations, Springer Series in Computational Mathematics, 23 Springer-Verlag

26. Rannacher, R.: (1992) On Chorin's projection method for the incompressible NavierStokes equations, Lectures Notes in Mathematics 1530, Springer, Berlin, 167-183

27. Temam, R.: (1977) Navier-Stokes Equations, Studies in Mathematics and its Applications, 2, North-Holland

28. Temam, R.: (1968) Une méthode d'approximation de la solution des équations de Navier-Stokes, Bull. Soc. Math. France 98, 115-152

Numerische Mathematik Electronic Edition page 238 of Numer. Math. (1998) 80: 207-238 